Submitted to Bernoulli

arXiv: arXiv: 1712.08058

\title{
Operator-scaling Gaussian random fields via aggregation
}

\author{
YI SHEN ${ }^{1}$ and YIZAO WANG ${ }^{2}$ \\ ${ }^{1}$ Department of Statistics and Actuarial Science, University of Waterloo, Mathematics 3 \\ Building, 200 University Avenue West Waterloo, Ontario N2L 3G1, Canada. \\ E-mail: yi.shen@uwaterloo.ca \\ 2 Department of Mathematical Sciences, University of Cincinnati, 2815 Commons Way, \\ ML-0025, Cincinnati, OH, 45221-0025, USA. E-mail: yizao.wang@uc.edu
}

\begin{abstract}
We propose an aggregated random-field model, and investigate the scaling limits of the aggregated partial-sum random fields. In this model, each copy in the aggregation is a \pm 1 -valued random field built from two correlated one-dimensional random walks, the law of each determined by a random persistence parameter. A flexible joint distribution of the two parameters is introduced, and given the parameters the two correlated random walks are conditionally independent. For the aggregated random field, when the persistence parameters are independent, the scaling limit is a fractional Brownian sheet. When the persistence parameters are tail-dependent, characterized in the framework of multivariate regular variation, the scaling limit is more delicate, and in particular depends on the growth rates of the underlying rectangular region along two directions: at different rates different operator-scaling Gaussian random fields appear as the region area tends to infinity. In particular, at the so-called critical speed, a large family of Gaussian random fields with long-range dependence arise in the limit. We also identify four different regimes at non-critical speed where fractional Brownian sheets arise in the limit.
\end{abstract}

Keywords:, Gaussian random field, fractional Brownian sheet, operator-scaling property, functional central limit theorem, long-range dependence, aggregation.

\section{Introduction and main results}

Long-range dependence phenomena are well known in various areas of applications, including notably econometrics, finance, and network traffic modeling. It is also referred to as long memory, particularly in time-series setup. Traditionally, a stationary stochastic process with finite second moment is considered to have long-range dependence if, roughly speaking, either the covariance function has the power law decay, or the spectral density has a singularity at the origin. The two approaches are referred to in the literature as the time-domain approach and the frequency-domain approach, respectively. Recently, interpretations of long-range dependence in terms of limit theorems have become more and more popular: a stochastic model of interest may be viewed to have longrange dependence if, for example, when compared to a similar model with short-range dependence, the normalization in certain limit theorem for partial sums is of a different 
order. The other model in comparison here may be the same model but with a different choice of parameter, or a much simplified model for which the short-range dependence has been well understood. The anomalous normalization already indicates the qualitatively different behavior of the model. Moreover, a functional limit theorem provides a more precise description of the macroscopic dependence, in terms of the limit process, and new families of stochastic processes have been discovered in this way. At the same time, limit theorems also provide insightful explanation on how long-range dependence appears, and the limit process, due to the intriguing dependence structure inherited from the discrete model, may be of independent interest for further investigation. Excellent references on long-range dependence in stochastic processes and applications include for example $[2,26,31]$.

In the investigation of long-range dependence, two classes of models have prominent roles: models via aggregation and models via filtration (often in the form of fractionally integrated processes or random fields). We shall focus on aggregated models in this paper. One of the most famous aggregated models is due to Robinson [30] and Granger [11], who showed that the aggregation of autoregressive processes with random parameters may lead to long memory. In particular, this model has received huge success in explaining the long memory phenomena in many economics and financial data sets in the econometrics literature. Another area where aggregated models have been extensively investigated is modeling long memory in network traffic. See for example [13, 22, 23].

In the spatial setup, however, aggregated random fields have been much less developed than their one-dimensional counterparts. See for example Lavancier $[14,16]$ and references therein. In particular, we are interested in aggregated spatial models of which, if scaled appropriately, the limit random fields are operator-scaling Gaussian random fields. We say a random field $\left\{\mathbb{G}_{t}\right\}_{t \geq 0}$ is operator-scaling, if for some $\beta_{1}, \beta_{2}, H>0$ we have

$$
\left\{\mathbb{G}_{\lambda^{\beta_{1}} t_{1}, \lambda^{\beta_{2}} t_{2}}\right\}_{\boldsymbol{t} \geq \mathbf{0}} \stackrel{\mathrm{d}}{=} \lambda^{H}\left\{\mathbb{G}_{\boldsymbol{t}}\right\}_{\boldsymbol{t} \geq \mathbf{0}}, \text { for all } \lambda>0
$$

This is actually a special case of the operator-scaling property introduced by Biermé et al. [6]. This property is an extension of the well-known self-similar property for onedimensional stochastic processes. Most operator-scaling random fields are anisotropic in the sense that they have different scaling properties in different directions, a very desirable property from modeling point of view. At the same time, this property also makes the analysis of such Gaussian random fields very challenging, and they have attracted much research interest since its introduction. See for example $[18,21,35,36]$ for recent developments on path properties of operator-scaling Gaussian random fields. Most operator-scaling random fields, as their one-dimensional counterparts, exhibit long-range dependence. Gaussian random fields with long-range dependence are known to have applications in medical image processing [7, 19] and hydrology [1, 20]. Econometric interpretation for aggregated models has also been discussed in the literature [17]. In terms of limit theorems, not many models that scale to anisotropic operator-scaling Gaussian random fields have been known, including notably [4, 5, 9, 15, 16, 24, 27, 28, 34]. Among these, only Lavancier [16] and Puplinskaitè and Surgailis [28] considered certain aggregated random fields (not strictly in our sense though, see Remark 1.7), while only

imsart-bj ver. 2014/10/16 file: aggregation20190726BEJ_combined.tex date: July 29, 2019 
Puplinskaitè and Surgailis [28] considered anisotropic aggregated ones.

In this paper, we propose a new aggregated random-field model that scales to a large family of anisotropic operator-scaling Gaussian random fields. Our model may be viewed as an extension of the approximation of fractional Brownian motions by aggregated random walks introduced by Enriquez [10]. In fact, Enriquez [10] proposed two different models for approximation of fractional Brownian motions with Hurst index $H>1 / 2$ and $H<1 / 2$ respectively, and our extension is based on the one for $H>1 / 2$ here. In this case, the Enriquez model can be viewed as an aggregation of independent copies of correlated random walks, where the law of each correlated random walk is completely determined by a random persistence parameter. We will investigate in another paper the extension of the other Enriquez model (for $H<1 / 2$ ), which is of a different nature.

In particular, our model inherited a prominent feature from the one-dimensional model that, in the aggregated model, each independent copy of the random field (or stochastic process in one dimension) takes only \pm 1 -values. It is appealing to restrict the values of model to \pm 1 from numerical simulation point of view. It also provides better insight on the dependence structure. Besides [10], a few recent limit theorems for \pm 1 -valued discrete models with long-range dependence include $[5,8,9,12]$.

The extension to random fields, however, is by no means straightforward. For each random field in the aggregation we are now searching for \pm 1 -valued models with non-trivial anisotropic dependence. The key idea is to consider two independent one-dimensional random walks as in the Enriquez model, and define the random field as the product of the two sequences of \pm 1 -valued steps of each; the dependence of the so-obtained random field is then determined by assigning an appropriate tail-dependence structure of the two persistence parameters (and keeping the random walks conditionally independent). Our modeling of the tail dependence is flexible, so that a large family of random fields arise in the limit, and also computable, so that we have explicit form of the asymptotic covariance of the limit Gaussian field, which is in general much more complex than in one dimension.

Below, we first review the Enriquez model in dimension one, and then introduce our generalization. The main results are then presented in Section 1.3.

\subsection{Enriquez model in dimension one}

Enriquez [10] proposed two aggregated models that scale to fractional Brownian motions, with Hurst index $H \in(1 / 2,1)$ and $H \in(0,1 / 2)$ respectively. We shall focus exclusively on the first one and its generalization to random fields, and we refer to this one as the Enriquez model in this paper, for the sake of simplicity.

The Enriquez model consists of aggregation of a family of independent $\{ \pm 1\}$-valued stationary sequences, with a parameter $H \in(1 / 2,1)$. The model is as follows. First, a random variable $q$ is sampled from the probability distribution $\mu_{H}$ on $(0,1)$ defined as

$$
\mu_{H}(d q)=(1-H) 2^{3-2 H}(1-q)^{1-2 H} \mathbf{1}_{\{q \in(1 / 2,1)\}} d q .
$$

For the sake of convenience, with a slight abuse of notation we let $q$ denote both a random variable in general and the variable in the density formula. Then, a sequence of random

imsart-bj ver. 2014/10/16 file: aggregation20190726BEJ_combined.tex date: July 29, 2019 
variables $\left\{\varepsilon_{n}\right\}_{n \in \mathbb{N}}$ is sampled iteratively: $\varepsilon_{1}$ is a $\{ \pm 1\}$-valued symmetric random variable, and for each $n \in \mathbb{N}$ given the past and $q, \varepsilon_{n+1}$ is set to take the same value of $\varepsilon_{n}$ with probability $q$, and the opposite with probability $1-q$. The law of the so-sampled sequence $\left\{\varepsilon_{n}\right\}_{n \in \mathbb{N}}$ is determined by, given $q$ and $\varepsilon_{1}$,

$$
\mathbb{P}\left(\varepsilon_{n+1}=1 \mid \varepsilon_{1}, \ldots, \varepsilon_{n}, q\right)=q \mathbf{1}_{\left\{\varepsilon_{n}=1\right\}}+(1-q) \mathbf{1}_{\left\{\varepsilon_{n}=-1\right\}}, n \in \mathbb{N} .
$$

Let $S_{n}:=\varepsilon_{1}+\cdots+\varepsilon_{n}$ denote the partial sum of the stationary sequence. For each $q$ fixed, the sequence $\left\{S_{n}\right\}_{n \in \mathbb{N}}$ can be viewed as a correlated $\{ \pm 1\}$-valued random walk, and $q$ is referred to as the persistence of the random walk. The partial-sum process $\left\{S_{n}(t)\right\}_{t \in[0,1]}$ of this sequence is denoted by

$$
S_{n}(t):=\sum_{j=1}^{\lfloor n t\rfloor} \varepsilon_{j}, t \in[0,1], n \in \mathbb{N} .
$$

Next, consider i.i.d. copies of the stationary sequence $\varepsilon$, each copy denoted by $\varepsilon^{i} \equiv$ $\left\{\varepsilon_{n}^{i}\right\}_{n \in \mathbb{N}}$. Let $\left\{S_{n}^{i}(t)\right\}_{t \in[0,1]}$ denote the partial-sum processes of the $i$-th sequence. Let $\{m(n)\}_{n \in \mathbb{N}}$ denote a increasing sequence of integers, and $\widehat{S}_{n}(t)$ denote the aggregated partial-sum process of $m(n)$ i.i.d. sequences

$$
\widehat{S}_{n}(t):=\sum_{i=1}^{m(n)} S_{n}^{i}(t)=\sum_{i=1}^{m(n)} \sum_{j=1}^{\lfloor n t\rfloor} \varepsilon_{j}^{i}
$$

Enriquez [10, Corollary 1] proved that if $\lim _{n \rightarrow \infty} m(n) / n^{2-2 H}=\infty$, then

$$
\left\{\frac{\widehat{S}_{n}(t)}{n^{H}}\right\}_{t \in[0,1]} \Rightarrow \sqrt{\frac{\Gamma(3-2 H)}{H(2 H-1)}}\left\{\mathbb{B}_{t}^{H}\right\}_{t \in[0,1]}
$$

in $D([0,1])$, where $\mathbb{B}^{H}$ is the fractional Brownian motion, a centered Gaussian process with covariance function

$$
\operatorname{Cov}\left(\mathbb{B}_{s}^{H}, \mathbb{B}_{t}^{H}\right)=\frac{1}{2}\left(s^{2 H}+t^{2 H}-|s-t|^{2 H}\right), s, t \geq 0 .
$$

\subsection{An aggregated random-field model}

We consider the following generalization of the Enriquez model, consisting of independent copies of a $\{ \pm 1\}$-valued stationary random field $\left\{X_{\boldsymbol{n}}\right\}_{\boldsymbol{n} \in \mathbb{N}^{2}}$. For each copy, a random vector $\boldsymbol{q}=\left(q_{1}, q_{2}\right)$ is first sampled from a certain distribution $\mu$ on $[1 / 2,1)^{2}$ to be described later. Next, given $q_{1}, q_{2} \in[1 / 2,1)$, let $\varepsilon^{(k)} \equiv\left\{\varepsilon_{n}^{(k)}\right\}_{n \in \mathbb{N}}, k=1,2$ be two conditionally independent one-dimensional random walks with persistence $q_{1}$ and $q_{2}$ respectively as in the original Enriquez model (each starting with $\mathbb{P}\left(\varepsilon_{1}^{(k)}= \pm 1\right)=1 / 2$ and following the dynamics determined by (1.3)). Then, consider the stationary random field

$$
X_{\boldsymbol{j}}:=\varepsilon_{j_{1}}^{(1)} \varepsilon_{j_{2}}^{(2)}, \boldsymbol{j} \in \mathbb{N}^{2} .
$$

imsart-bj ver. 2014/10/16 file: aggregation20190726BEJ_combined.tex date: July 29, 2019 
The stationarity of $X$ is easy to verify, regardless of the choice of $\mu$. Let

$$
S_{n}(t):=\sum_{j \in[1, n \cdot t]} X_{j}
$$

denote the partial sum of the random field. Here and below, $\boldsymbol{n} \cdot \boldsymbol{t}=\left(n_{1} t_{1}, n_{2} t_{2}\right) \in \mathbb{R}^{2}$ and $[\boldsymbol{a}, \boldsymbol{b}]$ is understood as

$$
[\boldsymbol{a}, \boldsymbol{b}] \equiv\left(\left[a_{1}, b_{1}\right] \times\left[a_{2}, b_{2}\right]\right) \cap \mathbb{Z}^{2}, \boldsymbol{a}, \boldsymbol{b} \in \mathbb{R}^{2}
$$

throughout the paper.

Next, let $\left\{X^{i}\right\}_{i \in \mathbb{N}}$ be i.i.d. copies of $X$, and define $S_{\boldsymbol{n}}^{i}(\boldsymbol{t})$ similarly as $S_{\boldsymbol{n}}(\boldsymbol{t})$. We then consider the aggregated partial-sum random field $\left\{\widehat{S}_{\boldsymbol{n}}(\boldsymbol{t})\right\}_{\boldsymbol{t} \in[0,1]^{2}}$ given by

$$
\widehat{S}_{\boldsymbol{n}}(\boldsymbol{t}):=\sum_{i=1}^{m(\boldsymbol{n})} S_{\boldsymbol{n}}^{i}(\boldsymbol{t}) \equiv \sum_{i=1}^{m(\boldsymbol{n})} \sum_{\boldsymbol{j} \in[\mathbf{1}, \boldsymbol{n} \cdot \boldsymbol{t}]} X_{\boldsymbol{j}}^{i}, \boldsymbol{t} \in[0,1]^{2},
$$

with $m(\boldsymbol{n}) \in \mathbb{N}$, the number of copies in the aggregation, to be chosen later.

Now we explain our choices of $\mu$, the law of $\boldsymbol{q}=\left(q_{1}, q_{2}\right)$. Recall that this is a probability measure on $[1 / 2,1)^{2}$. We consider two cases of the model with drastically different behaviors:

(i) independent persistence, where we assume that $q_{1}$ and $q_{2}$ are independent and with law $\mu_{H_{1}}$ and $\mu_{H_{2}}$, respectively. That is, $\mu=\mu_{H_{1}} \otimes \mu_{H_{2}}$. This is the easiest case of our limit theorems.

(ii) dependent persistence, where we assume that $q_{1}$ and $q_{2}$ are tail-dependent in the specific way described below. This is the case to which most of our effort is devoted.

In the case of dependent persistence, we introduce a specific and flexible model to characterize the tail dependence of $\boldsymbol{q}$ near $(1,1)$ as follows, which satisfies the multivariate regular variation assumption (see Remark 1.1 below). To start with, and for the convenience of analysis later, we construct a random vector $\boldsymbol{U} \in(0,1]^{2}$ with law $\mu^{*}$, and set $\mu$ as its induced measure on $[1 / 2,1)^{2}$ by

$$
\boldsymbol{q}=(1,1)-\frac{\boldsymbol{U}}{2}
$$

To allow flexible and analytically tractable dependence between $U_{1}$ and $U_{2}$, let $R$ be a positive continuous random variable with probability density $r^{-2} d r$ over $(1, \infty)$, and $\boldsymbol{W}=\left(W_{1}, W_{2}\right)$ be a random vector taking values in

$$
\Delta_{1}:=\left\{\boldsymbol{w} \in(0,1)^{2}: w_{1}+w_{2}=1\right\}
$$

with law $\Lambda$. We assume that $R$ and $\boldsymbol{W}$ are independent, and let $\alpha_{1}, \alpha_{2}$ be two constants in $(0,2)$. Then introduce

$$
\widetilde{U}_{k}:=\left(R W_{k}\right)^{-1 / \alpha_{k}}, k=1,2,
$$

imsart-bj ver. 2014/10/16 file: aggregation20190726BEJ_combined.tex date: July 29, 2019 
and set

$$
\boldsymbol{U}:= \begin{cases}\left(\widetilde{U}_{1}, \widetilde{U}_{2}\right) & \left(\widetilde{U}_{1}, \widetilde{U}_{2}\right) \in(0,1)^{2} \\ (1,1) & \text { otherwise }\end{cases}
$$

to address the practical issue that $U_{k}$ by definition should be in $[0,1]$. In this way, our aggregated random-field model with dependence parameters is completely determined by $\boldsymbol{\alpha}=\left(\alpha_{1}, \alpha_{2}\right)$ and $\Lambda$. In the dependent persistence case, all these parameters have impact on the limit random fields (see (1.11) below).

Remark 1.1. It is natural to work in the framework of multivariate regular variation for $\boldsymbol{q}$, as it is clear that for non-trivial dependence structure, only the behavior of $\boldsymbol{q}$ near $(1,1)$ matters: as an extension of the one-dimensional model we need each $q_{i}$ to have power-law density near 1 , and the new ingredient in two-dimensional modeling is to characterize the dependence of $\boldsymbol{q}$ at the tail $(1,1)$, a standard question in extreme value theory. However, for modeling the tail dependence, traditionally in extreme value theory and also in our application, it is more convenient to work with multivariate regular variation assumption at either $(\infty, \infty)$ or $(0,0)[29]$.

More precisely, for our application the tail dependence of $\boldsymbol{Z}=R \boldsymbol{W}$ at $(\infty, \infty)$ plays a crucial role in the limit (see (3.10) and (3.12)), which we model in the framework of multivariate regular variation in polar coordinate. A general assumption in this case should read as

$$
n \mathbb{P}\left(\left(\frac{\|\boldsymbol{Z}\|}{n}, \frac{\boldsymbol{Z}}{\|\boldsymbol{Z}\|}\right) \in \cdot\right) \stackrel{v}{\rightarrow} \frac{d r}{r^{2}} \times \Lambda(\cdot),
$$

$\|\boldsymbol{Z}\|:=\left|Z_{1}\right|+\left|Z_{2}\right|$, in the space of positive Radon measures on $[0, \infty]^{2} \backslash\{\mathbf{0}\}$ equipped with the vague topology, where $\stackrel{v}{\rightarrow}$ stands for the vague convergence, and $\Lambda$ is known as the angular measure that characterizes the tail dependence. Our construction of $R \boldsymbol{W}$ in (1.4) is a well known procedure that implies (1.6) [29, Section 6.5.3]. The advantage of working with $R \boldsymbol{W}$ directly instead of the weaker assumption (1.6) is to be able to obtain specific bounds quickly at various places, as the analysis is already quite involved.

\subsection{Main results}

Our main results are functional limit theorems on $\widehat{S}_{\boldsymbol{n}}(\boldsymbol{t})$. We first begin with the model with independent persistence.

Theorem 1.2. Consider the aggregated model with independent persistence $\left(\mu=\mu_{H_{1}} \otimes\right.$ $\mu_{H_{2}}, \mu_{H}$ as in $(1.2)$ and $\left.H_{1}, H_{2} \in(1 / 2,1)\right)$. Assume also

$$
\lim _{\boldsymbol{n} \rightarrow \infty} \frac{n_{1}^{2-2 H_{1}} n_{2}^{2-2 H_{2}}}{m(\boldsymbol{n})}=0 .
$$

Then,

$$
\frac{1}{n_{1}^{H_{1}} n_{2}^{H_{2}} \sqrt{m(\boldsymbol{n})}}\left\{\widehat{S}_{\boldsymbol{n}}(\boldsymbol{t})\right\}_{\boldsymbol{t} \in[0,1]^{2}} \Rightarrow \sigma\left\{\mathbb{B}_{\boldsymbol{t}}^{\boldsymbol{H}}\right\}_{\boldsymbol{t} \in[0,1]^{2}}
$$

imsart-bj ver. 2014/10/16 file: aggregation20190726BEJ_combined.tex date: July 29, 2019 
in $D\left([0,1]^{2}\right)$ as $\boldsymbol{n} \rightarrow \infty$, where $\mathbb{B}^{\boldsymbol{H}}$ is a standard fractional Brownian sheet with covariance function

$$
\operatorname{Cov}\left(\mathbb{B}_{\boldsymbol{s}}^{\boldsymbol{H}}, \mathbb{B}_{\boldsymbol{t}}^{\boldsymbol{H}}\right)=\prod_{k=1}^{2} \frac{1}{2}\left(s_{k}^{2 H_{k}}+t_{k}^{2 H_{k}}-\left|s_{k}-t_{k}\right|^{2 H_{k}}\right), \boldsymbol{s}, \boldsymbol{t} \geq 0
$$

and

$$
\sigma:=\prod_{k=1}^{2}\left(\frac{\Gamma\left(3-2 H_{k}\right)}{H_{k}\left(2 H_{k}-1\right)}\right)^{1 / 2}
$$

Here and below, more precisely, we actually consider a sequence of vectors $\{\boldsymbol{n}(j)\}_{j \in \mathbb{N}}$ in $\mathbb{N}^{2}$ and the limit as $j \rightarrow \infty$. It is always assumed that $\lim _{j \rightarrow \infty} n_{1}(j)=\infty$ and $\lim _{j \rightarrow \infty} n_{2}(j)=\infty$, so that the partial sum is over a rectangular region of which the lengths of both directions tend to infinity. For the sake of simplicity, throughout we drop the parameter $j$ and write $\boldsymbol{n} \rightarrow \infty$ instead of $j \rightarrow \infty$. We will also write $a(\boldsymbol{n}) \sim b(\boldsymbol{n})$ as $\boldsymbol{n} \rightarrow \infty$ if $\lim _{\boldsymbol{n} \rightarrow \infty} a(\boldsymbol{n}) / b(\boldsymbol{n})=1$.

For the model with dependent persistence, it turns out that the scaling limit depends on the relative growth rate of $n_{1}$ and $n_{2}$. We first look at partial sums over rectangles increasing at the so-called critical speed:

$$
n_{1}^{\alpha_{1}} \sim n_{2}^{\alpha_{2}} \text { as } \boldsymbol{n} \rightarrow \infty
$$

The following function

$$
\Psi_{\boldsymbol{\alpha}, \Lambda}(\boldsymbol{\theta}):=\int_{0}^{\infty} \int_{\Delta_{1}} \prod_{k=1}^{2} \frac{2\left(r w_{k}\right)^{-1 / \alpha_{k}}}{\left(r w_{k}\right)^{-2 / \alpha_{k}}+\theta_{k}^{2}} \Lambda(d \boldsymbol{w}) r^{-2} d r
$$

shows up in the harmonizable representation of the limit Gaussian random field. The finiteness of $\Psi_{\boldsymbol{\alpha}, \Lambda}$ will be established in (3.12) below.

Theorem 1.3. Consider the aggregated model with dependent persistence and $\alpha_{1}, \alpha_{2} \in$ $(0,2)$. If

$$
\lim _{\boldsymbol{n} \rightarrow \infty} \frac{n_{1}^{\alpha_{1}}}{m(\boldsymbol{n})}=0
$$

then, at critical speed (1.8),

$$
\frac{n^{\alpha_{1} / 2}}{|\boldsymbol{n}| \sqrt{m(\boldsymbol{n})}}\left\{\widehat{S}_{\boldsymbol{n}}(\boldsymbol{t})\right\}_{\boldsymbol{t} \in[0,1]^{2}} \Rightarrow\left\{\mathbb{G}_{\boldsymbol{t}}^{\boldsymbol{\alpha}, \Lambda}\right\}_{\boldsymbol{t} \in[0,1]^{2}}
$$

in $D\left([0,1]^{2}\right)$ as $\boldsymbol{n} \rightarrow \infty$, where $|\boldsymbol{n}|=n_{1} n_{2}$, and $\mathbb{G}^{\boldsymbol{\alpha}, \Lambda}$ is a centered Gaussian random field with

$$
\operatorname{Cov}\left(\mathbb{G}_{\boldsymbol{s}}^{\boldsymbol{\alpha}, \Lambda}, \mathbb{G}_{\boldsymbol{t}}^{\boldsymbol{\alpha}, \Lambda}\right)=\frac{1}{(2 \pi)^{2}} \int_{\mathbb{R}^{2}}\left(\prod_{k=1}^{2} \frac{\left(e^{i s_{k} \theta_{k}}-1\right) \overline{\left(e^{i t_{k} \theta_{k}}-1\right)}}{\left|\theta_{k}\right|^{2}}\right) \Psi_{\boldsymbol{\alpha}, \Lambda}(\boldsymbol{\theta}) d \boldsymbol{\theta} .
$$


Next, when $\boldsymbol{n}$ does not grow at the critical speed (1.8), we identify four different regimes. By symmetry, it suffices to assume

$$
n_{1}^{\alpha_{1}} \gg n_{2}^{\alpha_{2}}
$$

by which we mean $\lim _{n \rightarrow \infty} n_{2}^{\alpha_{2}} / n_{1}^{\alpha_{1}}=0$. Under this assumption the following theorem identifies two regimes of non-critical speed, and the other two regimes under the assumption $n_{1}^{\alpha_{1}} \ll n_{2}^{\alpha_{2}}$ can be read accordingly. In the sequel we write

$$
\mathfrak{c}_{H}:=B\left(H-\frac{1}{2}, \frac{3}{2}-H\right) \frac{\pi}{H \Gamma(2 H) \sin (H \pi)},
$$

where $B(a, b)=\int_{0}^{1} x^{a-1}(1-x)^{b-1} d x$ is the Beta function.

Theorem 1.4. Consider the aggregated model with dependent persistence and $\alpha_{1}, \alpha_{2} \in$ $(0,2)$. If

$$
\lim _{\boldsymbol{n} \rightarrow \infty} \frac{n_{1}^{2-2 H_{1}} n_{2}^{2-2 H_{2}}}{m(\boldsymbol{n})}=0
$$

then at non-critical speed (1.12),

$$
\frac{1}{n_{1}^{H_{1}} n_{2}^{H_{2}} \sqrt{m(\boldsymbol{n})}}\left\{\widehat{S}_{\boldsymbol{n}}(\boldsymbol{t})\right\}_{\boldsymbol{t} \in[0,1]^{2}} \Rightarrow \sigma\left\{\mathbb{B}_{\boldsymbol{t}}^{\boldsymbol{H}}\right\}_{\boldsymbol{t} \in[0,1]^{2}}
$$

in $D\left([0,1]^{2}\right)$ as $\boldsymbol{n} \rightarrow \infty$, where $\mathbb{B}^{\boldsymbol{H}}$ is the fractional Brownian sheet with Hurst indices $\boldsymbol{H}$, for the following two cases depending on the value of $\alpha_{1}$. In each case, $\boldsymbol{H}$ and $\sigma^{2}$ are given accordingly:

(i) $\alpha_{1}>1$ :

$$
H_{1}=\frac{1}{2}, H_{2}=1-\frac{\alpha_{2}}{2}\left(1-\frac{1}{\alpha_{1}}\right), \sigma^{2}=2 \alpha_{2} \mathfrak{c}_{H_{2}} \int_{\Delta_{1}} w_{1}^{1 / \alpha_{1}} w_{2}^{1-1 / \alpha_{1}} \Lambda(d \boldsymbol{w}),
$$

(ii) $\alpha_{1}<1$ :

$$
H_{1}=1-\frac{\alpha_{1}}{2}, H_{2}=1, \sigma^{2}=\alpha_{1} \mathfrak{c}_{H_{1}} \int_{\Delta_{1}} w_{1} \Lambda(d \boldsymbol{w}) .
$$

In the regimes of non-critical speed, the limit Gaussian random fields are fractional Brownian sheets that have a direction with degenerate dependence, in the sense that the Hurst index in that direction is either $1 / 2$ (independent increments) or 1 (complete dependence).

Remark 1.5. For the boundary case between the two regimes of non-critical speed in Theorem 1.4, namely $n_{1}^{\alpha_{1}} \gg n_{2}^{\alpha_{2}}$ and $\alpha_{1}=1$, we expect the following functional central limit theorem to hold

$$
\frac{1}{\sqrt{n_{1} \log n_{1}} n_{2} \sqrt{m(\boldsymbol{n})}}\left\{\widehat{S}_{\boldsymbol{n}}(\boldsymbol{t})\right\}_{\boldsymbol{t} \in[0,1]^{2}} \Rightarrow \sigma\left\{\mathbb{B}_{\boldsymbol{t}}^{\boldsymbol{H}}\right\}_{\boldsymbol{t} \in[0,1]^{2}},
$$


with $\boldsymbol{H}=(1 / 2,1)$ and $\sigma^{2}=4 \pi \int_{\Delta_{1}} w_{1} \Lambda(d \boldsymbol{w})$. Note that when compared to the two regimes therein, while there is the continuous transition in terms of the Hurst indices $\boldsymbol{H}$, the normalization is inconsistent with the one in (1.13), because of the extra logarithmic term. The analysis of this case is the most involved. However, in view of the limit, this is also the least interesting case as the limit random field has degenerate dependence in both directions. Therefore, we only prove the convergence of covariance function for (1.14) in the last section of the Supplementary Material.

All the random fields in the limit are operator-scaling. For fractional Brownian sheet, it is well known that

$$
\left\{\mathbb{B}_{\boldsymbol{\lambda} \cdot \boldsymbol{t}}^{\boldsymbol{H}}\right\}_{\boldsymbol{t} \geq \mathbf{0}} \stackrel{\mathrm{d}}{=} \lambda_{1}^{H_{1}} \lambda_{2}^{H_{2}}\left\{\mathbb{B}_{\boldsymbol{t}}^{\boldsymbol{H}}\right\}_{\boldsymbol{t} \geq \mathbf{0}},
$$

which is actually stronger than the operator-scaling property in (1.1). The limit random field $\mathbb{G}^{\boldsymbol{\alpha}, \Lambda}$ in Theorem 1.3 is also operator-scaling.

Proposition 1.6. For $\left\{\mathbb{G}_{\boldsymbol{t}}^{\boldsymbol{\alpha}, \Lambda}\right\}_{\boldsymbol{t} \geq \mathbf{0}}$ in Theorem 1.3, we have

$$
\left\{\mathbb{G}_{\lambda^{1 / \alpha_{1}} t_{1}, \lambda^{1 / \alpha_{2}} t_{2}}^{\boldsymbol{\alpha}, \Lambda}\right\}_{\boldsymbol{t} \geq \mathbf{0}} \stackrel{\mathrm{d}}{=} \lambda^{1 / \alpha_{1}+1 / \alpha_{2}-1 / 2}\left\{\mathbb{G}_{t}^{\boldsymbol{\alpha}, \Lambda}\right\}_{\boldsymbol{t} \geq \mathbf{0}}, \text { for all } \lambda>0 .
$$

Proof. Since $\left\{\mathbb{G}_{t}^{\boldsymbol{\alpha}, \Lambda}\right\}_{t \geq 0}$ is a Gaussian random field, it suffices to show

$$
\operatorname{Cov}\left(\mathbb{G}_{\lambda^{1 / \alpha_{1}} s_{1}, \lambda^{1 / \alpha_{2}} s_{2}}^{\boldsymbol{\alpha}, \Lambda}, \mathbb{G}_{\lambda^{1 / \alpha_{1}} t_{1}, \lambda^{1 / \alpha_{2}} t_{2}}^{\boldsymbol{\alpha}, \Lambda}\right)=\lambda^{2 / \alpha_{1}+2 / \alpha_{2}-1} \operatorname{Cov}\left(\mathbb{G}_{\boldsymbol{s}}^{\boldsymbol{\alpha}, \Lambda}, \mathbb{G}_{\boldsymbol{t}}^{\boldsymbol{\alpha}, \Lambda}\right) .
$$

Define $\boldsymbol{\theta}^{\prime}=\left(\theta_{1}^{\prime}, \theta_{2}^{\prime}\right):=\left(\lambda^{1 / \alpha_{1}} \theta_{1}, \lambda^{1 / \alpha_{2}} \theta_{2}\right)$, then

$$
\prod_{k=1}^{2} \frac{\left(e^{i \lambda^{1 / \alpha_{k}} s_{k} \theta_{k}}-1\right) \overline{\left(e^{i \lambda^{1 / \alpha_{k}} t_{k} \theta_{k}}-1\right)}}{\left|\theta_{k}\right|^{2}}=\lambda^{2 / \alpha_{1}+2 / \alpha_{2}} \prod_{k=1}^{2} \frac{\left(e^{i s_{k} \theta_{k}^{\prime}}-1\right) \overline{\left(e^{i t_{k} \theta_{k}^{\prime}}-1\right)}}{\left|\theta_{k}^{\prime}\right|^{2}} .
$$

For the function $\Psi_{\boldsymbol{\alpha}, \Lambda}$, we have

$$
\Psi_{\boldsymbol{\alpha}, \Lambda}\left(\boldsymbol{\theta}^{\prime}\right)=\lambda^{1-1 / \alpha_{1}-1 / \alpha_{2}} \Psi_{\boldsymbol{\alpha}, \Lambda}(\boldsymbol{\theta})
$$

by change of variable $r \rightarrow \lambda r$. Applying these two identities to (1.11) completes the proof.

The proofs of our results are based on estimates of asymptotics of second and fourth moments of the partial sums of each single random field $S_{n}$. However, except for the model with independent persistence, our estimates are by a different method from the one used in [10] in one dimension. The method used there is essentially the time-domain approach for long-range dependence, relying on the analysis of regular variation of the covariance function and the Karamata's theorem. This approach, however, cannot be easily adapted to two dimensions. Instead, we take the frequency-domain approach by working with Fourier transforms of the random fields.

imsart-bj ver. 2014/10/16 file: aggregation20190726BEJ_combined.tex date: July 29, 2019 


\subsection{Discussions}

We conclude the introduction with a few remarks.

Remark 1.7. There are other types of limit theorems in the investigation of aggregated models. For ours, we can write

$$
\frac{1}{a(\boldsymbol{n}) \sqrt{m(\boldsymbol{n})}} \widehat{S}_{\boldsymbol{n}}(\boldsymbol{t})=\frac{1}{a(\boldsymbol{n})} \sum_{\boldsymbol{j} \in[\mathbf{1}, \boldsymbol{n} \cdot \boldsymbol{t}]} \frac{1}{\sqrt{m(\boldsymbol{n})}} \sum_{i=1}^{m(\boldsymbol{n})} X_{\boldsymbol{j}}^{i} .
$$

Especially in econometrics literature, often the aggregated model is referred to the limit random field $\left\{\mathfrak{X}_{\boldsymbol{j}}\right\}_{\boldsymbol{j} \in \mathbb{N}^{2}}$ in the weak convergence $m^{-1 / 2} \sum_{i=1}^{m} X_{\boldsymbol{j}}^{(i)} \Rightarrow \mathfrak{X}_{\boldsymbol{j}}, \boldsymbol{j} \in \mathbb{N}^{2}$, and the investigation of the long-range dependence of the aggregation concerns the behavior of the covariance function of $\mathfrak{X}$, or equivalently its spectral density near origin. One may then scale these aggregated random fields to obtain operator-scaling random fields indexed by $\boldsymbol{t} \in[0,1]^{2}$ via

$$
\frac{1}{a(\boldsymbol{n})} \sum_{\boldsymbol{j} \in[\mathbf{1}, \boldsymbol{n} \cdot \boldsymbol{t}]} \mathfrak{X}_{\boldsymbol{j}}
$$

by appropriate choice of $a(\boldsymbol{n})$. The limit theorems in the form of (1.16) is referred to as taking a double limit, as one lets the number of copies in aggregation tend to infinity first (as $m \rightarrow \infty$ ), and then the size of the lattice tend to infinity (as $\boldsymbol{n} \rightarrow \infty$ ). The limit theorems in the form of (1.15) is referred to as taking a single limit.

Enriquez [10] established actually limit theorems by taking both single limit and double limit for the one-dimensional model. We only worked out the single limit here, which is more demanding to establish. If we take the double limit for our aggregated model, we expect the limit random fields to remain the same in all cases in aforementioned theorems, as shown in one dimension in [10]. We are not aware of any other limit theorems for aggregated random fields for single limits.

Remark 1.8. Our aggregated random-field model can be viewed as with an infinitedimensional parameter $\Lambda$ on $\Delta_{1}$ and $\boldsymbol{\alpha} \in(0,2)^{2}$, and hence it leads to a large flexible family of operator-scaling Gaussian random fields. There are several recent limit theorems on operator-scaling Gaussian random fields. However, besides the fractional Brownian sheets, it is not easy to compare the limits from different models. This suggests that the counterparts of fractional Brownian motions in high dimensions are far from being unique, which is a challenge for investigation of long-range dependence in high dimensions.

For example, Biermé et al. [5] established limit theorems for another flexible family of operator-scaling Gaussian random fields, in the investigation of a different random-field model. The Gaussian random fields in the limit have covariance function

$$
\sigma^{2} \int_{\mathbb{R}^{2}}\left(\prod_{k=1}^{2} \frac{\left(e^{i s_{k} \theta_{k}}-1\right) \overline{\left(e^{i t_{k} \theta_{k}}-1\right)}}{\left|\theta_{k}\right|^{2}}\right) \frac{1}{(\log \psi(\boldsymbol{\theta}))^{2}} d \boldsymbol{\theta}
$$

imsart-bj ver. 2014/10/16 file: aggregation20190726BEJ_combined.tex date: July 29, 2019 
where $\psi$ is the logarithm of the characteristic function of certain multivariate stable distribution. Puplinskaite and Surgailis [28] proposed another aggregated random-field model (in the sense of taking a double limit as in Remark 1.7), which may lead to both Gaussian and non-Gaussian stable limits. However, when restricted to a fixed domain of attraction, their model is essentially determined by one parameter (see [28, Eq. (1.8)], where $\beta$ plays the similar role as $q$ in Enriquez's original model), and hence is less flexible than ours and the one in [5].

It is not immediately clear to us whether it is possible to relate limit Gaussian random fields in $[5,28]$ to ours, and we leave this question to further investigation.

Remark 1.9. Our statements are actually more general than those in the aforementioned papers, where the rates of the rectangular regions are essentially assumed in the form of $n_{2}=n_{1}^{\gamma}$ for different choices of $\gamma$. We expect that assumptions therein can be generalized to the slightly more relaxed type here.

Remark 1.10. Here we observe a scaling-transition phenomenon, that is, when the underlying rectangles of the partial-sum random fields grow at different speeds, different random fields may arise in the limit. Such a phenomenon has been known in a few limit theorems for random fields in the literature recently [5, 27, 28], while our result here is the first, to the best of our knowledge, to investigate the boundary case between regimes of non-critical speed. The scaling-transition phenomenon is essentially due to the fact that the covariance function of the limit Gaussian random field, say $C(s, t)$, does not factorize into product form $C_{1}\left(s_{1}, t_{1}\right) C_{2}\left(s_{2}, t_{2}\right)$ in general, with the only exception when the random field is a fractional Brownian sheet.

In the rest of the paper, we prove Theorems 1.2, 1.3 and 1.4 in Sections 2, 3 and 4, respectively. Some auxiliary proofs are left to the Supplementary Material.

\section{Proof of Theorem 1.2}

The proof of Theorem 1.2 is based on three estimates, which all are based on a single random field $S_{n}$, not the aggregated one $\widehat{S}_{n}$.

Lemma 2.1. Under the assumption of Theorem 1.2,

$$
\operatorname{Cov}\left(S_{\boldsymbol{n}}(\boldsymbol{s}), S_{\boldsymbol{n}}(\boldsymbol{t})\right) \sim \sigma_{1}^{2} n_{1}^{2 H_{1}} n_{2}^{2 H_{2}} \operatorname{Cov}\left(\mathbb{B}_{\boldsymbol{s}}^{\boldsymbol{H}}, \mathbb{B}_{\boldsymbol{t}}^{\boldsymbol{H}}\right)
$$

as $\boldsymbol{n} \rightarrow \infty$, and there exists a constant $C$ such that

$$
\mathbb{E} S_{\boldsymbol{n}}(\boldsymbol{t})^{2} \leq C n_{1}^{2 H_{1}} n_{2}^{2 H_{2}} t_{1}^{2 H_{1}} t_{2}^{2 H_{2}} \text { for all } \boldsymbol{n} \in \mathbb{N}^{2}, \boldsymbol{t} \in[0,1]^{2},
$$

and

$$
\mathbb{E} S_{\boldsymbol{n}}(\boldsymbol{t})^{4} \leq C n_{1}^{2 H_{1}+2} n_{2}^{2 H_{2}+2} \text { for all } \boldsymbol{n} \in \mathbb{N}^{2}, \boldsymbol{t} \in[0,1]^{2} .
$$


Proof. Observe that

$$
S_{\boldsymbol{n}}(\boldsymbol{t})=\sum_{\boldsymbol{j} \in[\mathbf{1}, \boldsymbol{n} \cdot \boldsymbol{t}]} X_{\boldsymbol{j}}=\sum_{j_{1}=1}^{\left\lfloor n_{1} t_{1}\right\rfloor} \varepsilon_{j_{1}}^{(1)} \sum_{j_{2}=1}^{\left\lfloor n_{2} t_{2}\right\rfloor} \varepsilon_{j_{2}}^{(2)}=S_{\left\lfloor n_{1} t_{1}\right\rfloor}^{(1)} S_{\left\lfloor n_{2} t_{2}\right\rfloor}^{(2)},
$$

where $S_{n}^{(k)}=\sum_{j=1}^{n} \varepsilon_{j}^{(k)}, k=1,2$ are independent. Then,

$$
\operatorname{Cov}\left(S_{\boldsymbol{n}}(\boldsymbol{s}), S_{\boldsymbol{n}}(\boldsymbol{t})\right)=\prod_{k=1}^{2} \operatorname{Cov}\left(S_{n_{k}}^{(k)}\left(s_{k}\right), S_{n_{k}}^{(k)}\left(t_{k}\right)\right),
$$

and $\mathbb{E} S_{n}^{r}(\boldsymbol{t})=\prod_{k=1}^{2} \mathbb{E} S_{n_{k}}^{(k)}\left(t_{k}\right)^{r}$. The corresponding estimates on $S^{(k)}, k=1,2$ have been obtained in [10]. More precisely, in the proof of Corollary 1 in [10], it was shown that

$$
\operatorname{Cov}\left(S_{n_{k}}^{(k)}\left(s_{k}\right), S_{n_{k}}^{(k)}\left(t_{k}\right)\right) \sim \frac{\Gamma\left(3-2 H_{k}\right)}{H_{k}\left(2 H_{k}-1\right)} n_{k}^{2 H_{k}} \operatorname{Cov}\left(\mathbb{B}_{s_{k}}^{H_{k}}, \mathbb{B}_{t_{k}}^{H_{k}}\right)
$$

and

$$
\mathbb{E} S_{n_{k}}^{(k)}\left(t_{k}\right)^{r}=O\left(\left(n_{k} t_{k}\right)^{r+\left(2 H_{k}-2\right)}\right), r \in 2 \mathbb{N} .
$$

Taking the products for $k=1,2$ finishes the proof.

Proof of Theorem 1.2. We first prove the convergence of finite-dimensional distributions. It suffices to show, for all $d \in \mathbb{N}, a_{1}, \ldots, a_{d} \in \mathbb{R}, \boldsymbol{t}_{1}, \ldots, \boldsymbol{t}_{d} \in \mathbb{R}_{+}^{2}$,

$$
\frac{1}{n_{1}^{H_{1}} n_{2}^{H_{2}} \sqrt{m(\boldsymbol{n})}} \sum_{w=1}^{d} a_{w} \widehat{S}_{\boldsymbol{n}}\left(\boldsymbol{t}_{w}\right) \Rightarrow \sigma_{1} \sum_{w=1}^{d} a_{w} \mathbb{B}_{\boldsymbol{t}_{w}}^{\boldsymbol{H}} .
$$

Observe that the right-hand side is a centered Gaussian random variable. At the same time, the left-hand side can be expressed as

$$
\frac{1}{\sqrt{m(\boldsymbol{n})}} \sum_{i=1}^{m(\boldsymbol{n})} \frac{1}{n_{1}^{H_{1}} n_{2}^{H_{2}}} \sum_{w=1}^{d} a_{w} S_{\boldsymbol{n}}^{i}\left(\boldsymbol{t}_{w}\right) .
$$

By Lindeberg-Feller central limit theorem for triangular arrays of i.i.d. random variables, to show (2.4) it suffices to show, for

$$
\begin{gathered}
Y_{\boldsymbol{n}}:=\frac{1}{n_{1}^{H_{1}} n_{2}^{H_{2}}} \sum_{w=1}^{d} a_{w} S_{\boldsymbol{n}}\left(\boldsymbol{t}_{w}\right) \\
\lim _{\boldsymbol{n} \rightarrow \infty} \operatorname{Var}\left(Y_{\boldsymbol{n}}\right)=\sigma_{1}^{2} \operatorname{Var}\left(\sum_{w=1}^{d} a_{w} \mathbb{B}_{\boldsymbol{t}_{w}}^{\boldsymbol{H}}\right)
\end{gathered}
$$

and

$$
\lim _{n \rightarrow \infty} \mathbb{E}\left(Y_{n}^{2} \mathbf{1}_{\left\{Y_{n}^{2}>m(n) \eta\right\}}\right)=0 \text { for all } \eta>0 .
$$


For (2.6), Write

$$
\operatorname{Var}\left(Y_{\boldsymbol{n}}\right)=\frac{1}{n_{1}^{2 H_{1}} n_{2}^{2 H_{2}}} \sum_{w=1}^{d} \sum_{w^{\prime}=1}^{d} a_{w} a_{w^{\prime}} \operatorname{Cov}\left(S_{\boldsymbol{n}}\left(\boldsymbol{t}_{w}\right), S_{\boldsymbol{n}}\left(\boldsymbol{t}_{w^{\prime}}\right)\right),
$$

and similarly for $\operatorname{Var}\left(\sum_{w=1}^{d} a_{w} \mathbb{B}_{\boldsymbol{t}_{w}}^{\boldsymbol{H}}\right)$. Then, (2.6) follows from (2.1).

Next, we prove (2.7). Observe that by Markov inequality and (2.3),

$$
\begin{aligned}
\mathbb{E}\left(Y_{\boldsymbol{n}}^{2} \mathbf{1}_{\left\{Y_{\boldsymbol{n}}^{2}>m(\boldsymbol{n}) \eta\right\}}\right) & \leq \frac{1}{m(\boldsymbol{n}) \eta} \mathbb{E} Y_{\boldsymbol{n}}^{4} \leq \frac{1}{m(\boldsymbol{n}) \eta}\left(\frac{1}{n_{1}^{H_{1}} n_{2}^{H_{2}}} \sum_{w=1}^{d}\left|a_{w}\right|\left(\mathbb{E} S_{\boldsymbol{n}}\left(\boldsymbol{t}_{w}\right)^{4}\right)^{1 / 4}\right)^{4} \\
& \leq \frac{C}{m(\boldsymbol{n}) \eta n_{1}^{4 H_{1}} n_{2}^{4 H_{2}}} \sum_{w=1}^{d}\left|a_{w}\right| n_{1}^{2 H_{1}+2} n_{2}^{2 H_{2}+2}=\frac{C}{\eta} \frac{n_{1}^{2-2 H_{1}} n_{2}^{2-2 H_{2}}}{m(\boldsymbol{n})} .
\end{aligned}
$$

Therefore, (2.7) is satisfied, under the assumption (1.7).

Next, we prove the tightness. By [3, Theorem 3 and remark afterwards], it suffices to show that there exist $p \in \mathbb{N}, \gamma_{1}, \gamma_{2}>1, C>0$ such that

$$
\mathbb{E}\left|\frac{\widehat{S}_{\boldsymbol{n}}(\boldsymbol{t})}{n_{1}^{H_{1}} n_{2}^{H_{2}} \sqrt{m(\boldsymbol{n})}}\right|^{2 p} \leq C t_{1}^{\gamma_{1}} t_{2}^{\gamma_{2}}, \text { for all } \boldsymbol{n} \in \mathbb{N}^{2}, \boldsymbol{t} \in \mathbb{R}_{+}^{2} .
$$

For this purpose, observe that

$$
\mathbb{E} \widehat{S}_{\boldsymbol{n}}(\boldsymbol{t})^{2}=m(\boldsymbol{n}) \mathbb{E} S_{\boldsymbol{n}}(\boldsymbol{t})^{2} \leq C m(\boldsymbol{n})\left(n_{1} t_{1}\right)^{2 H_{1}}\left(n_{2} t_{2}\right)^{2 H_{2}}
$$

because of (2.2). The tightness thus follows.

As the above proof shows, the functional central limit theorem is essentially based on the three estimates in Lemma 2.1. The functional central limit theorems for other models will be very similarly based on corresponding estimates moments. For the model with independent persistence, these estimates are almost immediate from the one-dimensional ones in [10]. However, for the model with dependent persistence, the one-dimensional estimates can no longer be used, and we have to take a completely different approach.

\section{Proof of Theorem 1.3}

Throughout, we restrict ourselves to the aggregated model with dependent persistence, with

$$
\alpha_{1}, \alpha_{2} \in(0,2)
$$

and that

$$
n^{*}:=n_{1}^{\alpha_{1}} \sim n_{2}^{\alpha_{2}} \text { as } \boldsymbol{n} \rightarrow \infty
$$


which we shall assume in this section without further mentioning. Some of our estimates are universal and do not depend on this assumption, and in this case we will say explicitly "for all $\boldsymbol{n} \in \mathbb{N}^{2}$ ". We write also

$$
p(\boldsymbol{\alpha})=\frac{1}{\alpha_{1}}+\frac{1}{\alpha_{2}}
$$

in the sequel.

We start with the computation of the asymptotic covariance.

Proposition 3.1. We have

$$
\lim _{n \rightarrow \infty} \frac{\operatorname{Cov}\left(S_{\boldsymbol{n}}(\boldsymbol{s}), S_{\boldsymbol{n}}(\boldsymbol{t})\right)}{|\boldsymbol{n}|^{2} / n^{*}}=\operatorname{Cov}\left(\mathbb{G}_{\boldsymbol{s}}^{\boldsymbol{\alpha}, \Lambda}, \mathbb{G}_{\boldsymbol{t}}^{\boldsymbol{\alpha}, \Lambda}\right)
$$

and there exists a constant $C$ such that

$$
\mathbb{E} S_{\boldsymbol{n}}(\boldsymbol{t})^{2} \leq C \frac{|\boldsymbol{n}|^{2}}{n^{*}}\left(t_{1} t_{2}\right)^{2-1 / p(\boldsymbol{\alpha})}
$$

for all $\boldsymbol{t} \in[0,1]^{2}$ such that $\lfloor\boldsymbol{n} \cdot \boldsymbol{t}\rfloor=\boldsymbol{n} \cdot \boldsymbol{t}$.

The two estimates above are obtained by computing the Fourier transforms of the covariance. For background on multidimensional Fourier transforms, see [25].

Let $r$ denote the covariance function of the stationary random field

$$
r(\ell)=\operatorname{Cov}\left(X_{1}, X_{1+\ell}\right),
$$

and $\widehat{r}(\boldsymbol{\theta}):=\sum_{\boldsymbol{\ell} \in \mathbb{Z}^{2}} r(\boldsymbol{\ell}) \exp (i\langle\boldsymbol{\ell} \cdot \boldsymbol{\theta}\rangle)$ its Fourier transform. Introduce the Fourier transform of the sequence $\left\{a_{j}\right\}_{j \in \mathbb{N}}=\left\{\mathbf{1}_{\{1 \leq j \leq n\}}\right\}_{j \in \mathbb{N}}$

$$
D_{n}(\theta):=\sum_{j=1}^{n} e^{i j \theta}
$$

and set

$$
D_{\boldsymbol{n}, \boldsymbol{s}, \boldsymbol{t}}(\boldsymbol{\theta}):=\prod_{k=1}^{2} D_{\left\lfloor n_{k} s_{k}\right\rfloor}\left(\theta_{k}\right) \overline{D_{\left\lfloor n_{k} t_{k}\right\rfloor}\left(\theta_{k}\right)} .
$$

Lemma 3.2. We have

$$
\operatorname{Cov}\left(S_{\boldsymbol{n}}(\boldsymbol{s}), S_{\boldsymbol{n}}(\boldsymbol{t})\right)=\frac{1}{(2 \pi)^{2}} \int_{(-\pi, \pi)^{2}} D_{\boldsymbol{n}, \boldsymbol{s}, \boldsymbol{t}}(\boldsymbol{\theta}) \widehat{r}(\boldsymbol{\theta}) d \boldsymbol{\theta}
$$

Proof. To see this, we first write

$$
\operatorname{Cov}\left(S_{\boldsymbol{n}}(\boldsymbol{s}), S_{\boldsymbol{n}}(\boldsymbol{t})\right)=\sum_{\boldsymbol{i} \in[\mathbf{1}, \boldsymbol{n} \cdot \boldsymbol{s}]} \sum_{\boldsymbol{j} \in[\mathbf{1}, \boldsymbol{n} \cdot \boldsymbol{t}]} \operatorname{Cov}\left(X_{\boldsymbol{i}}, X_{\boldsymbol{j}}\right)=\sum_{\boldsymbol{\ell} \in \mathbb{Z}^{2}} r(\ell) \sum_{\boldsymbol{j} \in \mathbb{Z}^{2}} \mathbf{1}_{\{\boldsymbol{j} \in[\mathbf{1}, \boldsymbol{n} \cdot \boldsymbol{s}], \boldsymbol{j}+\boldsymbol{\ell} \in[\mathbf{1}, \boldsymbol{n} \cdot \boldsymbol{t}]\}} .
$$


Introduce $a_{\boldsymbol{j}}=\mathbf{1}_{\{\boldsymbol{j} \in[\mathbf{1}, \boldsymbol{n} \cdot \boldsymbol{s}]\}}, b_{\boldsymbol{j}}=\mathbf{1}_{\{\boldsymbol{j} \in[\mathbf{1}, \boldsymbol{n} \cdot \boldsymbol{t}]\}}, \boldsymbol{j} \in \mathbb{Z}^{2}$, and let $\widehat{a}(\boldsymbol{\theta})$ and $\widehat{b}(\boldsymbol{\theta})$ denote their Fourier transforms, respectively. Then, for each $\ell \in \mathbb{Z}^{2}$, we have

$$
\sum_{j \in \mathbb{Z}^{2}} \mathbf{1}_{\{\boldsymbol{j} \in[\mathbf{1}, \boldsymbol{n} \cdot \boldsymbol{s}], \boldsymbol{j}+\ell \in[\mathbf{1}, \boldsymbol{n} \cdot \boldsymbol{t}]\}}=\sum_{\boldsymbol{j} \in \mathbb{Z}^{2}} a_{\boldsymbol{j}} b_{\boldsymbol{j}+\boldsymbol{\ell}},
$$

which is the $\boldsymbol{\ell}$-th coefficient of $\widehat{\widehat{a}(\boldsymbol{\theta}) \hat{b}}(\boldsymbol{\theta})$. We have that

$$
\widehat{a}(\boldsymbol{\theta})=\prod_{k=1}^{2} D_{\left\lfloor n_{k} s_{k}\right\rfloor}\left(\theta_{k}\right) \quad \text { and } \quad \widehat{b}(\boldsymbol{\theta})=\prod_{k=1}^{2} D_{\left\lfloor n_{k} t_{k}\right\rfloor}\left(\theta_{k}\right) .
$$

So by Parseval's theorem, (3.4) becomes

$$
\operatorname{Cov}\left(S_{\boldsymbol{n}}(\boldsymbol{s}), S_{\boldsymbol{n}}(\boldsymbol{t})\right)=\frac{1}{(2 \pi)^{2}} \int_{(-\pi, \pi)^{2}} \overline{\overline{\widehat{a}(\boldsymbol{\theta})} \widehat{b}(\boldsymbol{\theta})} \widehat{r}(\boldsymbol{\theta}) d \boldsymbol{\theta},
$$

which yields (3.3).

The next step is to apply a change of variables

$$
\boldsymbol{\theta} \rightarrow \frac{\boldsymbol{\theta}}{\boldsymbol{n}}:=\left(\frac{\theta_{1}}{n_{1}}, \frac{\theta_{2}}{n_{2}}\right)
$$

and hence to write

$$
\operatorname{Cov}\left(S_{\boldsymbol{n}}(\boldsymbol{s}), S_{\boldsymbol{n}}(\boldsymbol{t})\right)=\frac{1}{|\boldsymbol{n}|(2 \pi)^{2}} \int_{\boldsymbol{n} \cdot(-\pi, \pi)^{2}} D_{\boldsymbol{n}, \boldsymbol{s}, \boldsymbol{t}}(\boldsymbol{\theta} / \boldsymbol{n}) \widehat{r}(\boldsymbol{\theta} / \boldsymbol{n}) d \boldsymbol{\theta} .
$$

The two functions of the integrand can then be treated separately. The following results on $D_{n, s, t}$ are well known and provided here only for the sake of completeness. In the sequel, we write

$$
\mathbb{R}_{o}^{2}=(\mathbb{R} \backslash\{0\})^{2} .
$$

Lemma 3.3. In the notations above,

$$
\lim _{\boldsymbol{n} \rightarrow \infty} \frac{D_{\boldsymbol{n}, \boldsymbol{s}, \boldsymbol{t}}(\boldsymbol{\theta} / \boldsymbol{n})}{|\boldsymbol{n}|^{2}}=\prod_{k=1}^{2} \frac{\left(e^{i s_{k} \theta_{k}}-1\right) \overline{\left(e^{i t_{k} \theta_{k}}-1\right)}}{\left|\theta_{k}\right|^{2}} \text { for all } \boldsymbol{\theta} \in \mathbb{R}_{o}^{2}
$$

and

$$
\left|\frac{D_{\boldsymbol{n}, \boldsymbol{s}, \boldsymbol{t}}(\boldsymbol{\theta} / \boldsymbol{n})}{|\boldsymbol{n}|^{2}}\right| \leq \pi^{2} \prod_{k=1}^{2} \min \left\{s_{k} t_{k}, \frac{1}{\left|\theta_{k}\right|^{2}}\right\}, \text { for all } \boldsymbol{n} \in \mathbb{N}^{2},\left|\theta_{k}\right| \leq n_{k} \pi
$$

Proof. It is easy to show that

$$
\lim _{n \rightarrow \infty} \frac{1}{n} D_{\lfloor n t\rfloor}\left(\frac{\theta}{n}\right)=\frac{e^{i t \theta}-1}{i \theta},
$$

imsart-bj ver. 2014/10/16 file: aggregation20190726BEJ_combined.tex date: July 29, 2019 
and, because of $|\sin (x)| \geq 2|x| / \pi$ for $|x| \leq \pi / 2$, and $|\sin x| \leq \min (|x|, 1)$,

$$
\left|\frac{1}{n} D_{\lfloor n t\rfloor}\left(\frac{\theta}{n}\right)\right|=\left|\frac{\sin (\lfloor n t\rfloor \theta /(2 n))}{n \sin (\theta /(2 n))}\right| \leq \pi \min \left\{t, \frac{1}{|\theta|}\right\}, n \in \mathbb{N},|\theta| \leq n \pi .
$$

The desired results now follow.

Most of the effort will be devoted to the analysis of $r$ and $\widehat{r}$.

Lemma 3.4. For $\boldsymbol{\theta} \in(-\pi, \pi)^{2}$ such that $\theta_{1} \neq 0, \theta_{2} \neq 0$,

$$
\widehat{r}(\boldsymbol{\theta})=\int G^{*}(\boldsymbol{u}, \boldsymbol{\theta}) \mu^{*}(d \boldsymbol{u}) \text { with } G^{*}(\boldsymbol{u}, \boldsymbol{\theta}):=\prod_{k=1}^{2} \frac{u_{k}\left(2-u_{k}\right)}{u_{k}^{2}+2\left(1-u_{k}\right)\left(1-\cos \theta_{k}\right)} .
$$

Moreover,

$$
\widehat{r}(\boldsymbol{\theta} / \boldsymbol{n}) \sim \frac{|\boldsymbol{n}|}{n^{*}} \Psi_{\boldsymbol{\alpha}, \Lambda}(\boldsymbol{\theta})
$$

as $\boldsymbol{n} \rightarrow \infty$, and there exists a constant $C$ such that

$$
\widehat{r}(\boldsymbol{\theta} / \boldsymbol{n}) \leq C\left(\frac{|\boldsymbol{n}|}{n^{*}}\left|\theta_{1}\right|^{1 / p(\boldsymbol{\alpha})-1}+\frac{n_{1}}{n^{*}}\left|\theta_{1}\right|^{\alpha_{1}-1}+\frac{n_{2}}{n^{*}}\left|\theta_{2}\right|^{\alpha_{2}-1}+1\right) .
$$

for all $\boldsymbol{\theta} \in \mathbb{R}_{o}^{2}$.

Proof. We have

$$
\begin{aligned}
r(\ell) & =\mathbb{E}\left(X_{1} X_{1+\ell}\right)=\mathbb{E}\left(\varepsilon_{1}^{(1)} \varepsilon_{1+\ell_{1}}^{(1)} \varepsilon_{1}^{(2)} \varepsilon_{1+\ell_{2}}^{(2)}\right)=\mathbb{E}\left[\mathbb{E}\left(\varepsilon_{1}^{(1)} \varepsilon_{1+\ell_{1}}^{(1)} \mid q_{1}\right) \mathbb{E}\left(\varepsilon_{1}^{(2)} \varepsilon_{1+\ell_{2}}^{(2)} \mid q_{2}\right)\right] \\
& =\mathbb{E}\left[\left(2 q_{1}-1\right)^{\left|\ell_{1}\right|}\left(2 q_{2}-1\right)^{\left|\ell_{2}\right|}\right]=\int\left(1-u_{1}\right)^{\left|\ell_{1}\right|}\left(1-u_{2}\right)^{\left|\ell_{2}\right|} \mu^{*}(d \boldsymbol{u}) .
\end{aligned}
$$

Consider

$$
\widehat{r}(\boldsymbol{\theta})=\sum_{\boldsymbol{\ell} \in \mathbb{Z}^{2}} r(\boldsymbol{\ell}) e^{i\langle\ell, \boldsymbol{\theta}\rangle}=\sum_{\boldsymbol{\ell} \in \mathbb{Z}^{2}} \int e^{i\langle\boldsymbol{\ell}, \boldsymbol{\theta}\rangle}\left(1-u_{1}\right)^{\left|\ell_{1}\right|}\left(1-u_{2}\right)^{\left|\ell_{2}\right|} \mu^{*}(d \boldsymbol{u}) .
$$

Recall that

$$
\sum_{\ell \in \mathbb{Z}} \rho^{|\ell|} e^{i \ell \theta}=\frac{1-\rho^{2}}{1-2 \rho \cos \theta+\rho^{2}}, \text { for all } \rho \in(-1,1) .
$$

So (3.8) follows.

Now we investigate the asymptotics of $\widehat{r}$. Recall that we let $\mu^{*}$ denote the measure on $(0,1]^{2}$ induced by $\boldsymbol{U}$. It turns out to be convenient to work with polar coordinates. For this purpose, introduce

$$
T_{\boldsymbol{\alpha}}(\boldsymbol{x}):=\left(\frac{1}{x_{1}^{\alpha_{1}}}, \frac{1}{x_{2}^{\alpha_{2}}}\right)
$$

imsart-bj ver. 2014/10/16 file: aggregation20190726BEJ_combined.tex date: July 29, 2019 
So $\mu^{*} \circ T_{\alpha}^{-1}$ is the measure on $[1, \infty)^{2}$ induced by $R \boldsymbol{W}$, and for any measurable function $f: \mathbb{R}_{+}^{2} \rightarrow \mathbb{R}$

$$
\int_{T_{\boldsymbol{\alpha}}\left((0,1]^{2}\right)} f(\boldsymbol{x}) \mu^{*} \circ T_{\boldsymbol{\alpha}}^{-1}(d \boldsymbol{x})=\int_{1}^{\infty} \int_{\Delta_{1}} \mathbf{1}_{\left\{r \boldsymbol{w} \in T_{\boldsymbol{\alpha}}\left((0,1]^{2}\right)\right\}} f(r \boldsymbol{w}) \Lambda(d \boldsymbol{w}) r^{-2} d r
$$

provided the integrability of either side can be justified. We treat $\boldsymbol{U}=(1,1)$ and $\boldsymbol{U} \in$ $(0,1)^{2}$ separately, and write

$$
\begin{aligned}
\widehat{r}(\boldsymbol{\theta} / \boldsymbol{n}) & =\int_{T_{\boldsymbol{\alpha}}\left((0,1]^{2}\right)} G^{*}\left(T_{\boldsymbol{\alpha}}^{-1}(\boldsymbol{u}), \boldsymbol{\theta} / \boldsymbol{n}\right) \mu^{*} \circ T_{\boldsymbol{\alpha}}^{-1}(d \boldsymbol{u})+\mathbb{P}(\boldsymbol{U}=(1,1)) \\
& =: \widehat{r}_{1}(\boldsymbol{\theta} / \boldsymbol{n})+\mathbb{P}(\boldsymbol{U}=(1,1)) .
\end{aligned}
$$

We shall see eventually that $\widehat{r}_{1}(\boldsymbol{\theta} / \boldsymbol{n})$ is of order $|\boldsymbol{n}| / n_{1}^{\alpha_{1}}$, so $\widehat{r}(\boldsymbol{\theta} / \boldsymbol{n}) \sim \widehat{r}_{1}(\boldsymbol{\theta} / \boldsymbol{n})$. We focus on $\widehat{r}_{1}(\boldsymbol{\theta} / \boldsymbol{n})$ from now on. Recall that $n^{*}=n_{1}^{\alpha_{1}}$. Note that

$$
\begin{aligned}
\widehat{r}_{1}(\boldsymbol{\theta} / \boldsymbol{n}) & =\int_{1}^{\infty} \int_{\Delta_{1}} \mathbf{1}_{\left\{r \boldsymbol{w} \in T_{\boldsymbol{\alpha}}\left((0,1]^{2}\right)\right\}} G^{*}\left(T_{\boldsymbol{\alpha}}^{-1}(r \boldsymbol{w}), \boldsymbol{\theta} / \boldsymbol{n}\right) \Lambda(d \boldsymbol{w}) r^{-2} d r \\
& =\frac{1}{n^{*}} \int_{0}^{\infty} \int_{\Delta_{1}} \mathbf{1}_{\left\{n^{*} r \boldsymbol{w} \in T_{\boldsymbol{\alpha}}\left((0,1]^{2}\right)\right\}} G^{*}\left(T_{\boldsymbol{\alpha}}^{-1}\left(n^{*} r \boldsymbol{w}\right), \boldsymbol{\theta} / \boldsymbol{n}\right) \Lambda(d \boldsymbol{w}) r^{-2} d r .
\end{aligned}
$$

In the last line above, we first applied a change of variables, and then replaced $\int_{1 / n^{*}}^{\infty}$ by $\int_{0}^{\infty}$, as the constraint $n^{*} r \boldsymbol{w} \in T_{\boldsymbol{\alpha}}\left((0,1)^{2}\right)$ implies that $r \geq\left(n^{*} w_{k}\right)^{-1} \geq\left(n^{*}\right)^{-1}$. Introduce

$$
h_{\boldsymbol{n}}(r, \boldsymbol{w}, \boldsymbol{\theta}):=G^{*}\left(T_{\boldsymbol{\alpha}}^{-1}\left(n^{*} r \boldsymbol{w}\right), \boldsymbol{\theta} / \boldsymbol{n}\right) \mathbf{1}_{\left\{n^{*} r \boldsymbol{w} \in T_{\boldsymbol{\alpha}}\left((0,1)^{2}\right)\right\}} .
$$

In view of integral expressions (1.9) and (3.11), to show the first part of the lemma we need to prove, for

$$
h(r, \boldsymbol{w}, \boldsymbol{\theta}):=\prod_{k=1}^{2} \frac{2\left(r w_{k}\right)^{-1 / \alpha_{k}}}{\left(r w_{k}\right)^{-2 / \alpha_{k}}+\theta_{k}^{2}},
$$

that

$$
\begin{aligned}
\frac{n^{*}}{|\boldsymbol{n}|} \widehat{r}_{1}(\boldsymbol{\theta} / \boldsymbol{n}) & \equiv \frac{1}{|\boldsymbol{n}|} \int_{0}^{\infty} \int_{\Delta_{1}} h_{\boldsymbol{n}}(r, \boldsymbol{w}, \boldsymbol{\theta}) \Lambda(d \boldsymbol{w}) r^{-2} d r \\
& \rightarrow \Psi_{\boldsymbol{\alpha}, \Lambda}(\boldsymbol{\theta}) \equiv \int_{0}^{\infty} \int_{\Delta_{1}} h(r, \boldsymbol{w}, \boldsymbol{\theta}) \Lambda(d \boldsymbol{w}) r^{-2} d r \in(0, \infty) \text { as } \boldsymbol{n} \rightarrow \infty .
\end{aligned}
$$

For this purpose, we show, for any $\delta \in(0,1)$,

$$
\begin{aligned}
\lim _{\boldsymbol{n} \rightarrow \infty} \frac{1}{|\boldsymbol{n}|} \int_{0}^{\infty} \int_{\Delta_{1}} h_{\boldsymbol{n}}(r, \boldsymbol{w}, \boldsymbol{\theta}) \mathbf{1}_{\left\{n^{*} r \boldsymbol{w} \in T_{\boldsymbol{\alpha}}\left((0, \delta]^{2}\right)\right\}} \Lambda(d \boldsymbol{w}) r^{-2} d r \\
=\int_{0}^{\infty} \int_{\Delta_{1}} h(r, \boldsymbol{w}, \boldsymbol{\theta}) \Lambda(d \boldsymbol{w}) r^{-2} d r
\end{aligned}
$$


and for some constant $C$ independent of $\boldsymbol{\theta}$,

$$
\begin{aligned}
& \int_{0}^{\infty} \int_{\Delta_{1}} h_{\boldsymbol{n}}(r, \boldsymbol{w}, \boldsymbol{\theta}) \mathbf{1}_{\left\{n^{*} r \boldsymbol{w} \in T_{\boldsymbol{\alpha}}\left((0,1]^{2} \backslash(0, \delta]^{2}\right)\right\}} \Lambda(d \boldsymbol{w}) r^{-2} d r \\
& \leq C\left(n_{1}\left|\theta_{1}\right|^{\alpha_{1}-1}+n_{2}\left|\theta_{2}\right|^{\alpha_{2}-1}+n^{*}\right) .
\end{aligned}
$$

Thus the integral in (3.14) does not contribute in the limit, since $|\boldsymbol{n}| \sim\left(n^{*}\right)^{p(\boldsymbol{\alpha})}$ as $\boldsymbol{n} \rightarrow \infty$ and $p(\boldsymbol{\alpha})>1$.

We first show (3.13). By the definition of $G^{*}$, we have

$$
\begin{aligned}
h_{\boldsymbol{n}, \delta}(r, \boldsymbol{w}, \boldsymbol{\theta}) & :=h_{\boldsymbol{n}}(r, \boldsymbol{w}, \boldsymbol{\theta}) \mathbf{1}_{\left\{n^{*} r \boldsymbol{w} \in T_{\boldsymbol{\alpha}}\left((0, \delta]^{2}\right)\right\}}=G^{*}\left(T_{\boldsymbol{\alpha}}^{-1}\left(n^{*} r \boldsymbol{w}\right), \boldsymbol{\theta} / \boldsymbol{n}\right) \mathbf{1}_{\left\{n^{*} r \boldsymbol{w} \in T_{\boldsymbol{\alpha}}\left((0, \delta]^{2}\right)\right\}} \\
& =\prod_{k=1}^{2} g\left(\left(n^{*} r w_{k}\right)^{-1 / \alpha_{k}}, \theta_{k} / n_{k}\right) \mathbf{1}_{\left\{n^{*} r \boldsymbol{w} \in T_{\boldsymbol{\alpha}}\left((0, \delta]^{2}\right)\right\}}
\end{aligned}
$$

with

$$
g(u, \theta):=\frac{u(2-u)}{u^{2}+2(1-u)(1-\cos \theta)} .
$$

It is clear that for every $r>0, \boldsymbol{w} \in \Delta_{1}, \boldsymbol{\theta} \in \mathbb{R}_{o}^{2}$,

$$
\lim _{\boldsymbol{n} \rightarrow \infty} \frac{1}{|\boldsymbol{n}|} h_{\boldsymbol{n}}(r, \boldsymbol{w}, \boldsymbol{\theta})=h(r, \boldsymbol{w}, \boldsymbol{\theta}) .
$$

So to prove (3.13), by the dominated convergence theorem it remains to find an integrable upper bound of $h_{\boldsymbol{n}, \delta} /|\boldsymbol{n}|$. For this purpose, observe that by the trivial bound $g(u, \theta) \leq$ $2 u^{-1}$,

$$
g\left(\left(n^{*} r w\right)^{-1 / \alpha_{k}}, \theta_{k} / n_{k}\right) \leq 2\left(n^{*} r w_{k}\right)^{1 / \alpha_{k}},
$$

and that, recalling the fact $2(1-\cos \theta)=4 \sin ^{2}(\theta / 2) \geq 4 \theta^{2} / \pi^{2}$ for $\theta \in(-\pi, \pi)$,

$$
g\left(\left(n^{*} r w_{k}\right)^{-1 / \alpha_{k}}, \theta_{k} / n_{k}\right) \mathbf{1}_{\left\{\left(n^{*} r w_{k}\right)^{\left.-1 / \alpha_{k} \in(0, \delta)\right\}}\right.} \leq \frac{\left(n^{*} r w_{k}\right)^{-1 / \alpha_{k}}}{2(1-\delta) \theta_{k}^{2} n_{k}^{-2} / \pi^{2}}=\frac{C_{\delta} n_{k}}{\left(r w_{k}\right)^{1 / \alpha_{k}} \theta_{k}^{2}}
$$

for some constant $C_{\delta}$ depending only on $\delta$. Here we used the fact that there exists universal constants $c_{1}, c_{2}$ such that $c_{1} n_{2}^{\alpha_{2}} \leq n^{*} \leq c_{2} n_{2}^{\alpha_{2}}$ for the sequence $\boldsymbol{n}$ of our interest. Therefore,

$$
\frac{1}{|\boldsymbol{n}|} h_{\boldsymbol{n}, \delta}(r, \boldsymbol{w}, \boldsymbol{\theta}) \leq C_{\delta} \prod_{k=1}^{2} \min \left\{\left(r w_{k}\right)^{1 / \alpha_{k}}, \frac{1}{\left(r w_{k}\right)^{1 / \alpha_{k}} \theta_{k}^{2}}\right\}=: C_{\delta} \bar{h}(r, \boldsymbol{w}, \boldsymbol{\theta}) .
$$

We now show that $\iint \bar{h}(r, \boldsymbol{w}, \boldsymbol{\theta}) \Lambda(d \boldsymbol{w}) r^{-2} d r<\infty$. Introduce

$$
d(\boldsymbol{\theta}, \boldsymbol{w}, \boldsymbol{\alpha}):=\left|\theta_{1} \theta_{2}\right|^{-1 / p(\boldsymbol{\alpha})} w_{1}^{-\alpha_{2} /\left(\alpha_{1}+\alpha_{2}\right)} w_{2}^{-\alpha_{1} /\left(\alpha_{1}+\alpha_{2}\right)} .
$$

imsart-bj ver. 2014/10/16 file: aggregation20190726BEJ_combined.tex date: July 29, 2019 
Then

$$
\begin{aligned}
\iint \bar{h}(r, \boldsymbol{w}, \boldsymbol{\theta}) \Lambda(d \boldsymbol{w}) r^{-2} d r \leq & \int_{\Delta_{1}} \int_{0}^{d(\boldsymbol{\theta}, \boldsymbol{w}, \boldsymbol{\alpha})} r^{p(\boldsymbol{\alpha})-2} d r w_{1}^{1 / \alpha_{1}} w_{2}^{1 / \alpha_{2}} \Lambda(d \boldsymbol{w}) \\
& +\int_{\Delta_{1}} \int_{d(\boldsymbol{\theta}, \boldsymbol{w}, \boldsymbol{\alpha})}^{\infty} r^{-p(\boldsymbol{\alpha})-2} d r w_{1}^{-1 / \alpha_{1}} w_{2}^{-1 / \alpha_{2}} \frac{1}{\left|\theta_{1} \theta_{2}\right|} \Lambda(d \boldsymbol{w}) .
\end{aligned}
$$

It can be easily verified that each double integral on the right-hand side above is bounded by

$$
C\left|\theta_{1} \theta_{2}\right|^{1 / p(\boldsymbol{\alpha})-1} \int_{\Delta_{1}} w_{1}^{\alpha_{2} /\left(\alpha_{1}+\alpha_{2}\right)} w_{2}^{\alpha_{1} /\left(\alpha_{1}+\alpha_{2}\right)} \Lambda(d \boldsymbol{w}) \leq C\left|\theta_{1} \theta_{2}\right|^{1 / p(\boldsymbol{\alpha})-1} .
$$

Namely, there exists a constant $C$ depending only on $\Lambda, \alpha_{1}, \alpha_{2}$, such that

$$
\int_{\mathbb{R}_{+} \times \Delta_{1}} \bar{h}(r, \boldsymbol{w}, \boldsymbol{\theta}) \Lambda(d \boldsymbol{w}) r^{-2} d r \leq C\left|\theta_{1} \theta_{2}\right|^{1 / p(\boldsymbol{\alpha})-1} .
$$

So we have proved (3.13) and

$$
\int_{0}^{\infty} \int_{\Delta_{1}} h_{\boldsymbol{n}, \delta}(r, \boldsymbol{w}, \boldsymbol{\theta}) \Lambda(d \boldsymbol{w}) r^{-2} d r \leq C|\boldsymbol{n}|\left|\theta_{1} \theta_{2}\right|^{1 / p(\boldsymbol{\alpha})-1} .
$$

Now we prove (3.14). We shall divide the region $\left\{n^{*} r \boldsymbol{w} \in T_{\boldsymbol{\alpha}}\left((0,1]^{2} \backslash(0, \delta]^{2}\right)\right\}$ into three pieces and treat each corresponding integral respectively. First, for $u \in(\delta, 1]$, we have $g(u, \theta) \leq 2 / \delta^{2}$, and hence

$$
h_{\boldsymbol{n}}(r, \boldsymbol{w}, \boldsymbol{\theta}) \mathbf{1}_{\left\{n^{*} r \boldsymbol{w} \in T_{\boldsymbol{\alpha}}\left((\delta, 1]^{2}\right)\right\}} \leq C_{\delta} .
$$

Thus,

$$
\begin{aligned}
& \int_{0}^{\infty} \quad \int_{\Delta_{1}} h_{\boldsymbol{n}}(r, \boldsymbol{w}, \boldsymbol{\theta}) \mathbf{1}_{\left\{n^{*} r \boldsymbol{w} \in T_{\boldsymbol{\alpha}}\left((\delta, 1]^{2}\right)\right\}} \Lambda(d \boldsymbol{w}) r^{-2} d r \\
& \quad \leq C_{\delta} \int_{\Delta_{1}} \int_{\left(n^{*}\right)^{-1}\left(w_{1}^{-1} \vee w_{2}^{-1}\right)}^{\left(n^{*}\right)^{-1}\left[\left(w_{1} \delta^{\alpha_{1}}\right)^{-1} \wedge\left(w_{2} \delta^{\alpha_{2}}\right)^{-1}\right]} r^{-2} d r \Lambda(d \boldsymbol{w}) \leq C_{\delta} n^{*} \int_{\Delta_{1}} w_{1} \wedge w_{2} \Lambda(d \boldsymbol{w}) \\
& \quad \leq C_{\delta} n^{*}
\end{aligned}
$$

Similarly,

$$
\begin{aligned}
h_{\boldsymbol{n}}(r, \boldsymbol{w}, \boldsymbol{\theta}) \mathbf{1}_{\left\{n^{*} r \boldsymbol{w} \in T_{\boldsymbol{\alpha}}((0, \delta] \times(\delta, 1])\right\}} & \leq C_{\delta} g\left(\left(n^{*} r w_{1}\right)^{-1 / \alpha_{1}}, \frac{\theta_{1}}{n_{1}}\right) \mathbf{1}_{\left\{n^{*} r w_{1} \geq \delta^{-\alpha_{1}}\right\}} \\
& \leq C_{\delta} n_{1} \min \left\{\left(r w_{1}\right)^{1 / \alpha_{1}}, \frac{1}{\left(r w_{1}\right)^{1 / \alpha_{1}} \theta_{1}^{2}}\right\} .
\end{aligned}
$$

This time, taking $d_{1}(\theta, w, \alpha):=|\theta|^{-\alpha} w^{-1}$, and writing

$$
\int_{\Delta_{1}} \int_{0}^{\infty}=\int_{\Delta_{1}}\left(\int_{0}^{d_{1}\left(\theta_{1}, w_{1}, \alpha_{1}\right)}+\int_{d_{1}\left(\theta_{1}, w_{1}, \alpha_{1}\right)}^{\infty}\right)
$$

imsart-bj ver. 2014/10/16 file: aggregation20190726BEJ_combined.tex date: July 29, 2019 
we have

$$
\int_{0}^{\infty} \int_{\Delta_{1}} h_{\boldsymbol{n}}(r, \boldsymbol{w}, \boldsymbol{\theta}) \mathbf{1}_{\left\{n^{*} r \boldsymbol{w} \in T_{\boldsymbol{\alpha}}((0, \delta] \times(\delta, 1])\right\}} \Lambda(d \boldsymbol{w}) r^{-2} d r \leq C_{\delta} n_{1}\left|\theta_{1}\right|^{\alpha_{1}-1} \int_{\Delta_{1}} w_{1} \Lambda(d \boldsymbol{w}) .
$$

By symmetry, a similar bound holds for the left-hand side above with the indicator function replaced by $\mathbf{1}_{\left\{n^{*} r \boldsymbol{w} \in T_{\alpha}((\delta, 1] \times(0, \delta])\right\}}$. Now (3.9) follows from combining (3.12), (3.17), (3.18) and (3.19).

Proof of Proposition 3.1. Recall (3.5). By Lemmas 3.3 and 3.4, the dominated convergence theorem yields the first part of the proposition, and that the integral in (1.11) is finite (recalling the assumption that $\alpha_{1}, \alpha_{2} \in(0,2)$ ). The details are omitted. For the second part, it also follows from Lemma 3.4, (3.5) and (3.6) that

$$
\begin{aligned}
\mathbb{E} S_{\boldsymbol{n}}(\boldsymbol{t})^{2} \leq C|\boldsymbol{n}| \int_{\boldsymbol{n} \cdot(-\pi, \pi)^{2}} & \prod_{k=1}^{2} \min \left\{t_{k}^{2}, \frac{1}{\left|\theta_{k}\right|^{2}}\right\} \\
& \times\left(\frac{|\boldsymbol{n}|}{n^{*}}\left|\theta_{1} \theta_{2}\right|^{1 / p(\boldsymbol{\alpha})-1}+\frac{n_{1}}{n^{*}}\left|\theta_{1}\right|^{\alpha_{1}-1}+\frac{n_{2}}{n^{*}}\left|\theta_{2}\right|^{\alpha_{2}-1}+1\right) d \boldsymbol{\theta} .
\end{aligned}
$$

By change of variables, the above integral is bounded by

$$
t_{1} t_{2} \int_{\mathbb{R}^{2}} \prod_{k=1}^{2} \min \left\{1, \frac{1}{\theta_{k}^{2}}\right\}\left(\frac{|\boldsymbol{n}|}{n^{*}}\left|\frac{\theta_{1} \theta_{2}}{t_{1} t_{2}}\right|^{1 / p(\boldsymbol{\alpha})-1}+\frac{n_{1}}{n^{*}}\left|\frac{\theta_{1}}{t_{1}}\right|^{\alpha_{1}-1}+\frac{n_{2}}{n^{*}}\left|\frac{\theta_{2}}{t_{2}}\right|^{\alpha_{2}-1}+1\right) d \boldsymbol{\theta} .
$$

Therefore, it follows that, for a constant $C$ independent of $n$ and $\boldsymbol{t}$,

$$
\mathbb{E} S_{\boldsymbol{n}}(\boldsymbol{t})^{2} \leq C\left(\frac{|\boldsymbol{n}|^{2}}{n^{*}}\left(t_{1} t_{2}\right)^{2-1 / p(\boldsymbol{\alpha})}+\frac{n_{1}|\boldsymbol{n}|}{n^{*}} t_{1}^{2-\alpha_{1}} t_{2}+\frac{n_{2}|\boldsymbol{n}|}{n^{*}} t_{1} t_{2}^{2-\alpha_{2}}+|\boldsymbol{n}| t_{1} t_{2}\right) .
$$

For $\boldsymbol{n} \in \mathbb{N}^{2}$ and $\boldsymbol{t} \in[0,1]^{2}$ such that $\boldsymbol{n} \cdot \boldsymbol{t}=\lfloor\boldsymbol{n} \cdot \boldsymbol{t}\rfloor$, we have

$$
\begin{aligned}
\frac{n_{1}|\boldsymbol{n}|}{n^{*}} t_{1}^{2-\alpha_{1}} t_{2} & =\frac{n_{1}^{2}}{n^{*}} t_{1}^{2-\alpha_{1}} \cdot n_{2} t_{2} \leq C\left(n_{1} t_{1}\right)^{2-\alpha_{1}}\left(n_{2} t_{2}\right)^{2-1 / p(\boldsymbol{\alpha})} \\
& \leq C\left(|\boldsymbol{n}| t_{1} t_{2}\right)^{2-1 / p(\boldsymbol{\alpha})} \leq C \frac{|\boldsymbol{n}|^{2}}{n^{*}}\left(t_{1} t_{2}\right)^{2-1 / p(\boldsymbol{\alpha})}
\end{aligned}
$$

and

$$
|\boldsymbol{n}| t_{1} t_{2} \leq|\boldsymbol{n}|^{2-1 / p(\boldsymbol{\alpha})}\left(t_{1} t_{2}\right)^{2-1 / p(\boldsymbol{\alpha})} \leq C \frac{|\boldsymbol{n}|^{2}}{n^{*}}\left(t_{1} t_{2}\right)^{2-1 / p(\boldsymbol{\alpha})},
$$

where we used the assumption (3.1). We have thus obtained the second part of the proposition.

The second estimate that we need is on the fourth moment. 
Proposition 3.5. There exists a constant $C$ such that

$$
\mathbb{E} S_{\boldsymbol{n}}(\boldsymbol{t})^{4} \leq C \frac{|\boldsymbol{n}|^{4}}{n^{*}}\left(t_{1} t_{2}\right)^{4-1 / p(\boldsymbol{\alpha})}
$$

for all $\boldsymbol{n} \in \mathbb{N}^{2}, \boldsymbol{t} \in[0,1]^{2}$, such that $\lfloor\boldsymbol{n} \cdot \boldsymbol{t}\rfloor=\boldsymbol{n} \cdot \boldsymbol{t}$.

Proof. Writing $\mathbb{E}_{q}(\cdot)=\mathbb{E}(\cdot \mid q)$, we have

$$
\mathbb{E} S_{\boldsymbol{n}}(\boldsymbol{t})^{4}=\mathbb{E}\left(\sum_{\boldsymbol{i} \in[\mathbf{1}, \boldsymbol{n} \cdot \boldsymbol{t}]} X_{\boldsymbol{i}}\right)^{4}=\mathbb{E}\left[\prod_{k=1}^{2} \mathbb{E}_{q_{k}}\left(\sum_{i_{k}=1}^{\left\lfloor n_{k} t_{k}\right\rfloor} \varepsilon_{i_{k}}^{(k)}\right)^{4}\right] .
$$

Note that, for $\left\{\varepsilon_{n}\right\}_{n \in \mathbb{N}}$ from the one-dimensional Enriquez model with random parameter $q, \mathbb{E}_{q} S_{n}^{4} \leq C \sum_{1 \leq i_{1} \leq \cdots \leq i_{4} \leq n} \mathbb{E}_{q}\left(\varepsilon_{i_{1}} \cdots \varepsilon_{i_{4}}\right)$, and

$$
\begin{aligned}
\sum_{1 \leq i_{1} \leq \cdots \leq i_{4} \leq n} \mathbb{E}_{q}\left(\varepsilon_{i_{1}} \cdots \varepsilon_{i_{4}}\right)=\sum_{\substack{j_{1}, j_{2} \geq 0, k_{1}, k_{2} \geq 0 \\
j_{1}+j_{2}+k_{1}+k_{2} \leq n-1}}(2 q-1)^{j_{1}+j_{2}} \\
=\sum_{\ell=0}^{n-1}(2 q-1)^{\ell} \sum_{\substack{j_{1}, j_{2} \geq 0 \\
j_{1}+j_{2}=\ell}} \sum_{\substack{k_{1}, k_{2} \geq 0 \\
k_{1}+k_{2} \leq n-1-\ell}} 1=\sum_{\ell=0}^{n-1}(2 q-1)^{\ell}\left(\begin{array}{c}
\ell+1 \\
1
\end{array}\right)\left(\begin{array}{c}
n+1-\ell \\
2
\end{array}\right) .
\end{aligned}
$$

So, for some constant $C$,

$$
\sum_{1 \leq i_{1} \leq \cdots \leq i_{4} \leq n} \mathbb{E}_{q}\left(\varepsilon_{i_{1}} \cdots \varepsilon_{i_{4}}\right) \leq C \sum_{\ell=-n}^{n}(2 q-1)^{|\ell|}|\ell|(n-|\ell|)^{2} .
$$

Thus,

$$
\begin{aligned}
\mathbb{E} S_{\boldsymbol{n}}(\boldsymbol{t})^{4} & \leq C \int \prod_{k=1}^{2} \sum_{\ell_{k}=-\left\lfloor n_{k} t_{k}\right\rfloor}^{\left\lfloor n_{k} t_{k}\right\rfloor}\left[\left|\ell_{k}\right|\left(\left\lfloor n_{k} t_{k}\right\rfloor-\left|\ell_{k}\right|\right)^{2}\left(2 q_{k}-1\right)^{\left|\ell_{k}\right|}\right] \mu(d \boldsymbol{q}) \\
& =C \int \sum_{\boldsymbol{\ell} \in[-\boldsymbol{n} \cdot \boldsymbol{t}, \boldsymbol{n} \cdot \boldsymbol{t}]} \prod_{k=1}^{2}\left[\left|\ell_{k}\right|\left(\left\lfloor n_{k} t_{k}\right\rfloor-\left|\ell_{k}\right|\right)^{2}\left(2 q_{k}-1\right)^{\left|\ell_{k}\right|}\right] \mu(d \boldsymbol{q}) .
\end{aligned}
$$

Introduce

$$
J_{\boldsymbol{n}}^{*}(\boldsymbol{\theta}):=\sum_{\boldsymbol{\ell} \in[-\boldsymbol{n}, \boldsymbol{n}]} \prod_{k=1}^{2}\left[\left|\ell_{k}\right|\left(n_{k}-\left|\ell_{k}\right|\right)^{2}\right] e^{i\langle\boldsymbol{\ell}, \boldsymbol{\theta}\rangle}=\prod_{k=1}^{2} J_{n_{k}}\left(\theta_{k}\right), \boldsymbol{n} \in \mathbb{N}^{2},
$$

with

$$
J_{n}(\theta):=\sum_{\ell=-n}^{n}|\ell|(n-|\ell|)^{2} e^{i \ell \theta}
$$

imsart-bj ver. 2014/10/16 file: aggregation20190726BEJ_combined.tex date: July 29, 2019 
In summary,

$$
\begin{aligned}
\mathbb{E} S_{\boldsymbol{n}}(\boldsymbol{t})^{4} & \leq C \sum_{\boldsymbol{\ell} \in[-\boldsymbol{n} \cdot \boldsymbol{t}, \boldsymbol{n} \cdot \boldsymbol{t}]} \prod_{k=1}^{2}\left[\left|\ell_{k}\right|\left(\left\lfloor n_{k} t_{k}\right\rfloor-\left|\ell_{k}\right|\right)^{2}\right] \int \prod_{k=1}^{2}\left(1-u_{k}\right)^{\left|\ell_{k}\right|} \mu^{*}(d \boldsymbol{u}) \\
& =\frac{C}{(2 \pi)^{2}} \int_{(-\pi, \pi)^{2}} J_{\lfloor\boldsymbol{n} \cdot \boldsymbol{t}\rfloor}^{*}(\boldsymbol{\theta}) \widehat{r}(\boldsymbol{\theta}) d \boldsymbol{\theta} \\
& =\frac{C}{|\boldsymbol{n}|(2 \pi)^{2}} \int_{\boldsymbol{n} \cdot(-\pi, \pi)^{2}} J_{\lfloor\boldsymbol{n} \cdot \boldsymbol{t}\rfloor}^{*}(\boldsymbol{\theta} / \boldsymbol{n}) \widehat{r}(\boldsymbol{\theta} / \boldsymbol{n}) d \boldsymbol{\theta} .
\end{aligned}
$$

Now we establish the following bound: for some constant $C$,

$$
\left|J_{\lfloor n t\rfloor}\left(\frac{\theta}{n}\right)\right| \leq C n^{4} t^{2} \min \left\{t^{2}, \frac{1}{|\theta|^{2}}\right\} \text { for all } n \in \mathbb{N}, t \in[0,1], \theta \in(-n \pi, n \pi) .
$$

Observe that this bound and Lemma 3.4 yield the desired result.

To show (3.22), write

$$
J_{n}(\theta)=2 \operatorname{Re}\left(W_{n}(\theta)\right) \quad \text { with } \quad W_{n}(\theta):=\sum_{\ell=1}^{n}(n-\ell)^{2} \ell e^{i \ell \theta} .
$$

So

$$
\begin{aligned}
W_{n}(\theta)-e^{i \theta} W_{n}(\theta) & =\sum_{\ell=1}^{n}\left[(n-\ell)^{2} \ell-(n-\ell+1)^{2}(\ell-1)\right] e^{i \ell \theta} \\
& =\sum_{\ell=1}^{n} \sum_{p_{1}=0}^{2} \sum_{p_{2}=0}^{2-p_{1}} c_{p_{1}, p_{2}} n^{p_{1}} \ell^{p_{2}} e^{i \ell \theta}
\end{aligned}
$$

for some constants $c_{p_{1}, p_{2}}$ independent of $n$ and $\theta$. Write $m=\lfloor n t\rfloor \leq n$. Then,

$$
\begin{aligned}
\left|J_{m}\left(\frac{\theta}{n}\right)\right| & \leq \frac{2}{\left|1-e^{i \theta / n}\right|}\left|\sum_{\ell=1}^{m} \sum_{p_{1}=0}^{2} \sum_{p_{2}=0}^{2-p_{1}} c_{p_{1}, p_{2}} m^{p_{1}} \ell^{p_{2}} e^{i \ell \theta / n}\right| \\
& \leq C \frac{n}{|\theta|} \sum_{p_{1}=0}^{2} m^{p_{1}} \sum_{p_{2}=0}^{2-p_{1}}\left|\sum_{\ell=1}^{m} \ell^{p_{2}} e^{i \ell \theta / n}\right|=C \frac{n}{|\theta|} \sum_{p_{1}=0}^{2} m^{p_{1}} \sum_{p_{2}=0}^{2-p_{1}}\left|V_{m, p_{2}+1}\left(\frac{\theta}{n}\right)\right|
\end{aligned}
$$

with

$$
V_{n, k}(\theta):=\sum_{\ell=1}^{n} \ell^{k-1} e^{i \ell \theta}
$$

Similarly as above, we have

$$
V_{n, k+1}(\theta)=\frac{1}{1-e^{i \theta}}\left(\sum_{\ell=1}^{n} \sum_{j=0}^{k-1} c_{j, k} \ell^{j} e^{i \ell \theta}-n^{k} e^{i(n+1) \theta}\right)
$$

imsart-bj ver. 2014/10/16 file: aggregation20190726BEJ_combined.tex date: July 29, 2019 
for some constants $c_{j, k}$. So

$$
\left|V_{m, k+1}\left(\frac{\theta}{n}\right)\right| \leq \frac{C n}{|\theta|}\left(\sum_{j=0}^{k-1}\left|\sum_{\ell=1}^{m} \ell^{j} e^{i \ell \theta / n}\right|+m^{k}\right)=\frac{C n}{|\theta|}\left(\sum_{j=1}^{k}\left|V_{m, j}\left(\frac{\theta}{n}\right)\right|+m^{k}\right) .
$$

At the same time, $\left|V_{m, k+1}(\theta / n)\right| \leq m^{k+1}$. We have seen in (3.7) that, for $m=\lfloor n t\rfloor \leq n$,

$$
\left|V_{m, 1}\left(\frac{\theta}{n}\right)\right| \leq n \pi \min \left\{\frac{m}{n}, \frac{1}{|\theta|}\right\}
$$

So by induction, we arrive at

$$
\left|V_{m, k+1}\left(\frac{\theta}{n}\right)\right| \leq C_{k} n m^{k} \min \left\{\frac{m}{n}, \frac{1}{|\theta|}\right\}, k \in \mathbb{N},
$$

where $C_{k}$ are constants depending on $k$. Hence by taking the maximum among $\left(C_{k}\right)_{k=0,1,2}$, we have

$$
\left|V_{m, k+1}\left(\frac{\theta}{n}\right)\right| \leq C n m^{k} \min \left\{\frac{m}{n}, \frac{1}{|\theta|}\right\}, k=0,1,2 .
$$

Applying this to (3.23) leads to

$$
\left|J_{m}\left(\frac{\theta}{n}\right)\right| \leq C \frac{n}{|\theta|} \sum_{p_{1}=0}^{2} m^{p_{1}} \sum_{p_{2}=0}^{2-p_{1}}\left(n m^{p_{2}}\right) \min \left\{\frac{m}{n}, \frac{1}{|\theta|}\right\} \leq C \frac{n^{2} m^{2}}{|\theta|} \min \left\{\frac{m}{n}, \frac{1}{|\theta|}\right\} .
$$

Note also that $\left|W_{m}(\theta)\right| \leq m^{4}$. We have thus proved (3.22). Then,

$$
J_{\lfloor\boldsymbol{n} \cdot \boldsymbol{t}\rfloor}^{*}(\boldsymbol{\theta} / \boldsymbol{n}) \leq C|\boldsymbol{n}|^{4}\left(t_{1} t_{2}\right)^{2} \prod_{k=1}^{2} \min \left\{t_{k}^{2}, \frac{1}{\left|\theta_{k}\right|^{2}}\right\} .
$$

Applying this and (3.9) to (3.21), one has

$$
\begin{aligned}
\mathbb{E} S_{\boldsymbol{n}}(\boldsymbol{t})^{4} \leq C|\boldsymbol{n}|^{3}\left(t_{1} t_{2}\right)^{2} \int \prod_{k=1}^{2} \min \left\{t_{k}^{2}, \frac{1}{\left|\theta_{k}\right|^{2}}\right\} \\
\quad \times\left(\frac{|\boldsymbol{n}|}{n^{*}}\left|\theta_{1} \theta_{2}\right|^{1 / p(\boldsymbol{\alpha})-1}+\frac{n_{1}}{n^{*}}\left|\theta_{1}\right|^{\alpha_{1}-1}+\frac{n_{2}}{n^{*}}\left|\theta_{2}\right|^{\alpha_{2}-1}+1\right) d \boldsymbol{\theta} \\
\leq C|\boldsymbol{n}|^{2}\left(t_{1} t_{2}\right)^{2} \cdot \frac{|\boldsymbol{n}|^{2}}{n^{*}}\left|t_{1} t_{2}\right|^{2-1 / p(\boldsymbol{\alpha})}
\end{aligned}
$$

where the upper bound for the integral has been treated as in (3.20). The desired result now follows.

Proof of Theorem 1.3. The proof follows the same line of the proof of Theorem 1.2. First we establish the finite-dimensional convergence by applying Lindeberg-Feller central limit theorem. The asymptotic covariance of the aggregated random field is the same 
as the asymptotic covariance of a single random field, up to appropriate normalization, since

$$
\operatorname{Cov}\left(\widehat{S}_{\boldsymbol{n}}(\boldsymbol{s}), \widehat{S}_{\boldsymbol{n}}(\boldsymbol{t})\right)=m(\boldsymbol{n}) \operatorname{Cov}\left(S_{\boldsymbol{n}}(\boldsymbol{s}), S_{\boldsymbol{n}}(\boldsymbol{t})\right) .
$$

The latter is established in Proposition 3.1. It remains to verify the counterpart here of the Lindeberg-Feller condition (2.7), which requires the fourth moment on $\mathbb{E} S_{\boldsymbol{n}}(\boldsymbol{t})^{4}$ established in Proposition 3.5. In this case we have, for

$$
\begin{gathered}
Y_{\boldsymbol{n}}:=\frac{1}{|\boldsymbol{n}|\left(n^{*}\right)^{-1 / 2}} \sum_{w=1}^{d} a_{w} S_{\boldsymbol{n}}\left(\boldsymbol{t}_{w}\right) \\
\mathbb{E}\left(Y_{\boldsymbol{n}}^{2} \mathbf{1}_{\left\{Y_{\boldsymbol{n}}^{2}>m(\boldsymbol{n}) \eta\right\}}\right) \leq \frac{\mathbb{E} Y_{\boldsymbol{n}}^{4}}{m(\boldsymbol{n}) \eta} \leq \frac{1}{m(\boldsymbol{n}) \eta}\left(\frac{\left(n^{*}\right)^{1 / 2}}{|\boldsymbol{n}|} \sum_{w=1}^{d}\left|a_{w}\right|\left(\mathbb{E} S_{\boldsymbol{n}}\left(\boldsymbol{t}_{w}\right)^{4}\right)^{1 / 4}\right)^{4} \\
\leq \frac{C\left(n^{*}\right)^{2}}{m(\boldsymbol{n}) \eta|\boldsymbol{n}|^{4}} \sum_{w=1}^{d}\left|a_{w}\right| \frac{|\boldsymbol{n}|^{4}}{n^{*}}=\frac{C}{\eta} \frac{n^{*}}{m(\boldsymbol{n})}
\end{gathered}
$$

which converges to 0 as $\boldsymbol{n} \rightarrow \infty$ for all $\eta>0$ under condition (1.10).

The tightness follows from (3.2), which implies the condition (2.8) introduced by Bickel and Wichura [3].

\section{Proof of Theorem 1.4}

We start by explaining how to identify the limits of each regime of non-critical speed, and the corresponding orders of the normalizations. Taken such information for granted, one could prove Theorem 1.4 directly by starting from the first section of the Supplementary Material. However, the identification of the four regimes (essentially two due to symmetry) are at the core of the problem, and we explain this step first. We also discuss the boundary case in the last section of the Supplementary Material.

Again we start with computing the asymptotic covariance, which shall indicate the normalization order and the limit Gaussian random field in each regime. We still apply the Fourier transform, and Lemma 3.2 still holds:

$$
\operatorname{Cov}\left(S_{\boldsymbol{n}}(\boldsymbol{s}), S_{\boldsymbol{n}}(\boldsymbol{t})\right)=\frac{1}{(2 \pi)^{2}} \int_{(-\pi, \pi)^{2}} D_{\boldsymbol{n}, \boldsymbol{s}, \boldsymbol{t}}(\boldsymbol{\theta}) \widehat{r}(\boldsymbol{\theta}) d \boldsymbol{\theta} .
$$

The evaluation of the asymptotics of the covariance in general depends on two changes of variables. First, introduce change of variables

$$
\boldsymbol{\theta} \rightarrow \frac{\boldsymbol{\theta}}{\boldsymbol{n}^{\prime}}:=\left(\frac{\theta_{1}}{n_{1}^{\prime}}, \frac{\theta_{2}}{n_{2}^{\prime}}\right) \quad \text { with } \quad \boldsymbol{n}^{\prime}=\left(n_{1}^{\prime}, n_{2}^{\prime}\right)
$$

imsart-bj ver. 2014/10/16 file: aggregation20190726BEJ_combined.tex date: July 29, 2019 
We have taken $\boldsymbol{n}^{\prime}=\boldsymbol{n}$ in the regime of critical speed. Here, however, we may need to pick $\boldsymbol{n}^{\prime}$ differently. So our starting point of analysis is the following expression of the covariance function of the random field:

$$
\operatorname{Cov}\left(S_{\boldsymbol{n}}(\boldsymbol{s}), S_{\boldsymbol{n}}(\boldsymbol{t})\right)=\frac{\left|\boldsymbol{n}^{\prime}\right|^{-1}}{(2 \pi)^{2}} \int_{\boldsymbol{n}^{\prime} \cdot(-\pi, \pi)^{2}} D_{\boldsymbol{n}, \boldsymbol{s}, \boldsymbol{t}}\left(\boldsymbol{\theta} / \boldsymbol{n}^{\prime}\right) \widehat{r}\left(\boldsymbol{\theta} / \boldsymbol{n}^{\prime}\right) d \boldsymbol{\theta} .
$$

Next, we take a closer look at $\widehat{r}\left(\boldsymbol{\theta} / \boldsymbol{n}^{\prime}\right)$. Recall

$$
g(u, \theta)=\frac{u(2-u)}{u^{2}+2(1-u)(1-\cos \theta)} .
$$

Then, we have, for $\boldsymbol{\theta} \in \boldsymbol{n}^{\prime} \cdot(-\pi, \pi)^{2}$,

$$
\begin{aligned}
\widehat{r}\left(\boldsymbol{\theta} / \boldsymbol{n}^{\prime}\right) & =\int_{\Delta_{1}} \int_{0}^{\infty} \prod_{k=1}^{2} g\left(\left(r w_{k}\right)^{-1 / \alpha_{k}}, \theta_{k} / n_{k}^{\prime}\right) \mathbf{1}_{\left\{r \boldsymbol{w} \in T_{\boldsymbol{\alpha}}\left((0,1]^{2}\right)\right\}} \frac{d r}{r^{2}} \Lambda(d \boldsymbol{w}) \\
& =\frac{1}{n^{*}} \int_{\Delta_{1}} \int_{0}^{\infty} \prod_{k=1}^{2} g\left(\left(n^{*} r w_{k}\right)^{-1 / \alpha_{k}}, \theta_{k} / n_{k}^{\prime}\right) \mathbf{1}_{\left\{n^{*} r \boldsymbol{w} \in T_{\boldsymbol{\alpha}}\left((0,1]^{2}\right)\right\}} \frac{d r}{r^{2}} \Lambda(d \boldsymbol{w}),
\end{aligned}
$$

where $n^{*}$ is a scalar factor satisfying $n^{*} \rightarrow \infty$ as $\boldsymbol{n} \rightarrow \infty$ (the rate to be discussed later), and the last step follows by the change of variables

$$
r \rightarrow n^{*} r
$$

So we can write the integral in (4.1) as a multiple integral over $\mathbb{R}^{2} \times \mathbb{R}_{+} \times \Delta_{1}$ with respect to the measure $d \boldsymbol{\theta} r^{-2} d r \Lambda(d \boldsymbol{w})$, with the integrand

$$
\begin{aligned}
\left(n^{*}\right)^{-1} \prod_{k=1}^{2} D_{\left\lfloor n_{k} s_{k}\right\rfloor}\left(\frac{\theta_{k}}{n_{k}^{\prime}}\right) \overline{D_{\left\lfloor n_{k} t_{k}\right\rfloor}\left(\frac{\theta_{k}}{n_{k}^{\prime}}\right)} \\
\quad \times \prod_{k=1}^{2} g\left(\left(n^{*} r w_{k}\right)^{-1 / \alpha_{k}}, \frac{\theta_{k}}{n_{k}^{\prime}}\right) \mathbf{1}_{\left\{n^{*} r \boldsymbol{w} \in T_{\boldsymbol{\alpha}}\left((0,1]^{2}\right)\right\}} \mathbf{1}_{\left\{\boldsymbol{\theta} \in \boldsymbol{n}^{\prime} \cdot(-\pi, \pi)^{2}\right\}} .
\end{aligned}
$$

As before, pointwise asymptotics of $D$ and $g$ are straightforward. We have

$$
\begin{aligned}
\lim _{n \rightarrow \infty}\left(n_{k}\right)^{-2} D_{\left\lfloor n_{k} s_{k}\right\rfloor}\left(\frac{\theta_{k}}{n_{k}^{\prime}}\right) \overline{D_{\left\lfloor n_{k} t_{k}\right\rfloor}\left(\frac{\theta_{k}}{n_{k}^{\prime}}\right)} & \\
= & \mathfrak{D}_{s_{k}, t_{k}}\left(\theta_{k}\right):= \begin{cases}\frac{\left(e^{i s_{k} \theta_{k}}-1\right) \overline{\left(e^{i t_{k} \theta_{k}}-1\right)}}{\left|\theta_{k}\right|^{2}} & n_{k}^{\prime} \sim n_{k} \\
s_{k} t_{k} & n_{k}^{\prime} \gg n_{k},\end{cases}
\end{aligned}
$$

and

$$
\lim _{n \rightarrow \infty} \frac{g\left(\left(n^{*} r\right)^{-1 / \alpha_{k}}, \theta_{k} / n_{k}^{\prime}\right)}{\left(n^{*}\right)^{1 / \alpha_{k}}}=\mathfrak{g}\left(r^{-1 / \alpha_{k}}, \theta_{k}\right):= \begin{cases}\frac{2 r^{-1 / \alpha_{k}}}{r^{-2 / \alpha_{k}}+\theta_{k}^{2}} & \left(n^{*}\right)^{1 / \alpha_{k}} \sim n_{k}^{\prime} \\ 2 r^{1 / \alpha_{k}} & \left(n^{*}\right)^{1 / \alpha_{k}} \ll n_{k}^{\prime} .\end{cases}
$$


We shall choose $n_{1}^{\prime}, n_{2}^{\prime}$ and $n^{*}$ as functions of $n_{1}$ or $n_{2}$. In this way, combining (4.1), (4.2), (4.3) and (4.4), we have, formally,

$$
\begin{aligned}
& \lim _{n \rightarrow \infty} \frac{\left|\boldsymbol{n}^{\prime}\right| \operatorname{Cov}\left(S_{\boldsymbol{n}}(\boldsymbol{s}), S_{\boldsymbol{n}}(\boldsymbol{t})\right)}{|\boldsymbol{n}|^{2}\left(n^{*}\right)^{p(\boldsymbol{\alpha})-1}} \\
& \quad=\frac{1}{(2 \pi)^{2}} \int_{\Delta_{1}} \int_{0}^{\infty} \int_{\mathbb{R}^{2}} \prod_{k=1}^{2} \mathfrak{D}_{s_{k}, t_{k}}\left(\theta_{k}\right) \mathfrak{g}\left(\left(r w_{k}\right)^{-1 / \alpha_{k}}, \theta_{k}\right) d \boldsymbol{\theta} \frac{d r}{r^{2}} \Lambda(d \boldsymbol{w}),
\end{aligned}
$$

where the functions $\mathfrak{D}$ and $\mathfrak{g}$ depend on the choice of $\boldsymbol{n}^{\prime}$ and $n^{*}$, and we only computed the pointwise convergence of the multiple integral.

However, a careful examination shall tell quickly that not all choices of $\boldsymbol{n}^{\prime}$ and $n^{*}$ will make (4.5) a legitimate statement, as the multiple integral is not always well defined: so we need those such that the multiple integral in (4.5) is well defined, finite and strictly non-zero. The first natural case to be considered is when both $\mathfrak{D}$ and $\mathfrak{g}$ are not degenerate, corresponding to the regime of critical speed already addressed in Theorem 1.3, with

$$
\boldsymbol{n}^{\prime}=\boldsymbol{n}, n^{*} \sim n_{1}^{\alpha_{1}} \sim n_{2}^{\alpha_{2}} .
$$

Then, it is not hard to see that the only other legitimate integrands are

$$
\begin{aligned}
& \prod_{k=1}^{2} \frac{\left(e^{i s_{k} \theta_{k}}-1\right) \overline{\left(e^{i t_{k} \theta_{k}}-1\right)}}{\theta_{k}^{2}} \frac{2\left(r w_{1}\right)^{-1 / \alpha_{1}}}{\left(r w_{1}\right)^{-2 / \alpha_{1}}+\theta_{1}^{2}} 2\left(r w_{2}\right)^{1 / \alpha_{2}}, \\
& \prod_{k=1}^{2} \frac{\left(e^{i s_{k} \theta_{k}}-1\right) \overline{\left(e^{i t_{k} \theta_{k}}-1\right)}}{\theta_{k}^{2}} 2\left(r w_{1}\right)^{1 / \alpha_{1}} \frac{2\left(r w_{2}\right)^{-1 / \alpha_{2}}}{\left(r w_{2}\right)^{-2 / \alpha_{2}}+\theta_{2}^{2}}, \\
& \frac{\left(e^{i s_{1} \theta_{1}}-1\right) \overline{\left(e^{i t_{1} \theta_{1}}-1\right)}}{\theta_{1}^{2}} s_{2} t_{2} \prod_{k=1}^{2} \frac{2\left(r w_{k}\right)^{-1 / \alpha_{k}}}{\left(r w_{k}\right)^{-2 / \alpha_{k}}+\theta_{k}^{2}}, \\
& s_{1} t_{1} \frac{\left(e^{i s_{2} \theta_{2}}-1\right) \overline{\left(e^{i t_{2} \theta_{2}}-1\right)}}{\theta_{2}^{2}} \prod_{k=1}^{2} \frac{2\left(r w_{k}\right)^{-1 / \alpha_{k}}}{\left(r w_{k}\right)^{-2 / \alpha_{k}}+\theta_{k}^{2}},
\end{aligned}
$$

and they correspond to the following four conditions on $\boldsymbol{n}^{\prime}$ and $n^{*}$, respectively

$$
\begin{aligned}
& \boldsymbol{n}^{\prime}=\boldsymbol{n}, n^{*} \sim n_{1}^{\alpha_{1}}, n_{1}^{\alpha_{1}} \ll n_{2}^{\alpha_{2}}, \\
& \boldsymbol{n}^{\prime}=\boldsymbol{n}, n^{*} \sim n_{2}^{\alpha_{2}}, n_{1}^{\alpha_{1}} \gg n_{2}^{\alpha_{2}}, \\
& \boldsymbol{n}^{\prime} \sim\left(\left(n^{*}\right)^{1 / \alpha_{1}},\left(n^{*}\right)^{1 / \alpha_{2}}\right), n^{*} \sim n_{1}^{\alpha_{1}}, n_{1}^{\alpha_{1}} \gg n_{2}^{\alpha_{2}}, \\
& \boldsymbol{n}^{\prime} \sim\left(\left(n^{*}\right)^{1 / \alpha_{1}},\left(n^{*}\right)^{1 / \alpha_{2}}\right), n^{*} \sim n_{2}^{\alpha_{2}}, n_{1}^{\alpha_{1}} \ll n_{2}^{\alpha_{2}} .
\end{aligned}
$$

We shall also see later that, for each integrand above to be integrable, an extra assumption on $\boldsymbol{\alpha}$ is needed.

By symmetry, it suffices to focus on the case

$$
n_{1}^{\alpha_{1}} \gg n_{2}^{\alpha_{2}}
$$

imsart-bj ver. 2014/10/16 file: aggregation20190726BEJ_combined.tex date: July 29, 2019 
which from now on we assume. Two identities are needed in these regimes with noncritical speed. The first identity is on the covariance function of fractional Brownian motion (e.g. [32, Proposition 7.2.8])

$$
\int_{\mathbb{R}} \frac{\left(e^{i s \theta}-1\right) \overline{\left(e^{i t \theta}-1\right)}}{|\theta|^{1+2 H}} d \theta=2 \pi C_{H} \operatorname{Cov}\left(\mathbb{B}_{s}^{H}, \mathbb{B}_{t}^{H}\right), s, t>0, H \in(0,1)
$$

with

$$
C_{H}=\frac{\pi}{H \Gamma(2 H) \sin (H \pi)}
$$

The second is the following

$$
\begin{aligned}
\int_{0}^{\infty} \frac{r^{-\gamma}}{(r w)^{-2 / \alpha}+\theta^{2}} d r=\frac{\alpha}{2} B\left(H-\frac{1}{2}, \frac{3}{2}-H\right) \frac{w^{\gamma-1}}{|\theta|^{2 H-1}} \\
\quad \text { if } H:=\frac{3-\alpha(\gamma-1)}{2} \in(1 / 2,3 / 2)
\end{aligned}
$$

and otherwise the integral is infinite. Here $B(a, b)=\int_{0}^{1} x^{a-1}(1-x)^{b-1} d x$ is the beta function. Indeed, by change of variables, we have

$$
\begin{aligned}
\int_{0}^{\infty} \frac{r^{-\gamma}}{(r w)^{-2 / \alpha}+\theta^{2}} d r & =w^{\gamma-1}|\theta|^{\alpha(\gamma-1)-2} \int_{0}^{\infty} \frac{r^{-\gamma}}{r^{-2 / \alpha}+1} d r \\
& =\frac{w^{\gamma-1}}{|\theta|^{2-\alpha(\gamma-1)}} \frac{\alpha}{2} \int_{0}^{\infty} \frac{r^{-(1+(1-\gamma) \alpha / 2)}}{r+1} d r
\end{aligned}
$$

Recall also that $\int_{0}^{\infty}(1+u)^{-1} u^{-\beta} d u=B(\beta, 1-\beta)=\pi / \sin (\pi \beta)$ for all $\beta \in(0,1)$, and otherwise the integral is infinite. Combining the above yields (4.10).

We begin with the case (4.8), by formally integrating (4.6) with respect to $d \boldsymbol{\theta} r^{-2} d r \Lambda(d \boldsymbol{w})$. First, by (4.9),

$$
\int_{\mathbb{R}} \frac{\left(e^{i s_{1} \theta_{1}}-1\right) \overline{\left(e^{i t_{1} \theta_{1}}-1\right)}}{\left|\theta_{1}\right|^{2}} d \theta_{1}=2 \pi \operatorname{Cov}\left(\mathbb{B}_{s_{1}}^{1 / 2}, \mathbb{B}_{t_{1}}^{1 / 2}\right)
$$

Next, by (4.10),

$$
\int_{0}^{\infty} \frac{\left(r w_{1}\right)^{1 / \alpha_{1}}\left(r w_{2}\right)^{-1 / \alpha_{2}}}{\left(r w_{2}\right)^{-2 / \alpha_{2}}+\theta_{2}^{2}} \frac{d r}{r^{2}}=\frac{\alpha_{2}}{2} B\left(H_{2}-\frac{1}{2}, \frac{3}{2}-H_{2}\right) \frac{w_{1}^{1 / \alpha_{1}} w_{2}^{1-1 / \alpha_{1}}}{\left|\theta_{2}\right|^{2 H_{2}-1}},
$$

with

$$
H_{2}=1-\frac{\alpha_{2}}{2}\left(1-\frac{1}{\alpha_{1}}\right) \quad \text { provided } \quad \frac{\alpha_{2}}{2}\left(1-\frac{1}{\alpha_{1}}\right) \in(0,1 / 2) .
$$

So the above formal calculation yields an extra necessary assumption $\alpha_{1}>1$ for the case (4.8), and in this case integrating (4.6) with respect to $d \boldsymbol{\theta} r^{-2} d r \Lambda(d \boldsymbol{w})$ yields, with 
$H_{1}=1 / 2$ and $H_{2}$ as in (4.12),

$$
\begin{aligned}
& (2 \pi)^{2} \int_{\Delta_{1}} w_{1}^{1 / \alpha_{1}} w_{2}^{1-1 / \alpha_{1}} \Lambda(d \boldsymbol{w}) 2 \alpha_{2} B\left(H_{2}-\frac{1}{2}, \frac{3}{2}-H_{2}\right) C_{H_{2}} \operatorname{Cov}\left(\mathbb{B}_{\boldsymbol{s}}^{\boldsymbol{H}}, \mathbb{B}_{\boldsymbol{t}}^{\boldsymbol{H}}\right) \\
& \quad=(2 \pi)^{2} 2 \alpha_{2} \mathfrak{c}_{H_{2}} \int_{\Delta_{1}} w_{1}^{1 / \alpha_{1}} w_{2}^{1-1 / \alpha_{1}} \Lambda(d \boldsymbol{w}) \operatorname{Cov}\left(\mathbb{B}_{\boldsymbol{s}}^{\boldsymbol{H}}, \mathbb{B}_{\boldsymbol{t}}^{\boldsymbol{H}}\right)=(2 \pi)^{2} \sigma^{2} \operatorname{Cov}\left(\mathbb{B}_{\boldsymbol{s}}^{\boldsymbol{H}}, \mathbb{B}_{\boldsymbol{t}}^{\boldsymbol{H}}\right)
\end{aligned}
$$

with $\sigma$ as in regime (i) in Theorem 1.4.

Now we identify the regime (ii). This time, the multiple integral on the right-hand side of (4.5) becomes (by integrating (4.7))

$$
\begin{aligned}
\int_{\Delta_{1}} \int_{0}^{\infty} & \int_{\mathbb{R}^{2}} \frac{\left(e^{i s_{1} \theta_{1}}-1\right) \overline{\left(e^{i t_{1} \theta_{1}}-1\right)}}{\left|\theta_{1}\right|^{2}} s_{2} t_{2} \prod_{k=1}^{2} \frac{2\left(r w_{k}\right)^{-1 / \alpha_{k}}}{\left(r w_{k}\right)^{-2 / \alpha_{k}}+\theta_{k}^{2}} \frac{d r}{r^{2}} d \boldsymbol{\theta} \Lambda(d \boldsymbol{w}) \\
= & 2 \pi\left(s_{2} t_{2}\right) \int_{\mathbb{R}} \frac{\left(e^{i s_{1} \theta_{1}}-1\right) \overline{\left(e^{i t_{1} \theta_{1}}-1\right)}}{\left|\theta_{1}\right|^{2}} \int_{\Delta_{1}} \int_{\mathbb{R}} \frac{2\left(r w_{1}\right)^{-1 / \alpha_{1}}}{\left(r w_{1}\right)^{-2 / \alpha_{1}}+\theta_{1}^{2}} \frac{d r}{r^{2}} \Lambda(d \boldsymbol{w}) d \theta_{1} .
\end{aligned}
$$

Again by (4.10), for

$$
H_{1}=1-\frac{\alpha_{1}}{2}, H_{2}=1 \quad \text { provided } \quad \alpha_{1} \in(0,1),
$$

the above becomes

$$
\begin{aligned}
& 2 \pi\left(s_{2} t_{2}\right) \alpha_{1} B\left(H_{1}-\frac{1}{2}, \frac{3}{2}-H_{1}\right) \int_{\Delta_{1}} w_{1} \Lambda(d \boldsymbol{w}) \int_{\mathbb{R}} \frac{\left(e^{i s_{1} \theta_{1}}-1\right) \overline{\left(e^{i t_{1} \theta_{1}}-1\right)}}{\left|\theta_{1}\right|^{1+2 H_{1}}} d \theta_{1} \\
&=(2 \pi)^{2} \alpha_{1} \mathfrak{c}_{H_{1}} \int_{\Delta_{1}} w_{1} \Lambda(d \boldsymbol{w}) \operatorname{Cov}\left(\mathbb{B}_{\boldsymbol{s}}^{\boldsymbol{H}}, \mathbb{B}_{\boldsymbol{t}}^{\boldsymbol{H}}\right) .
\end{aligned}
$$

This is the regime (ii).

To complete the computation of asymptotic covariance (4.5), it remains to provide an integrable bound to apply the dominated convergence theorem. To establish the limit theorem, we need to also bound the fourth-moment. These are left to the first two sections in the Supplementary Material.

\section{Supplementary Material}

Proof of Theorem 1.4 and the boundary case

(doi: COMPLETED BY THE TYPESETTER; .pdf). We prove the two regimes in Theorem 1.4 and the convergence of covariance in the boundary case for non-critical speed.

\section{Acknowledgement}

The authors thank two anonymous referees for careful reading and helpful comments. YS's research was supported in part by the Natural Sciences and Engineering Research Council of Canada (RGPIN-2014-04840). YW's research was supported in part by NSA grant H98230-16-1-0322 and Army Research Laboratory grant W911NF-17-1-0006.

imsart-bj ver. 2014/10/16 file: aggregation20190726BEJ_combined.tex date: July 29, 2019 


\section{References}

[1] Benson, D. A., Meerschaert, M. M., Baeumer, B., and Scheffler, H.-P. (2006). Aquifer operator scaling and the effect on solute mixing and dispersion. Water Resources Research, 42(1).

[2] Beran, J., Feng, Y., Ghosh, S., and Kulik, R. (2013). Long-memory processes. Springer, Heidelberg. Probabilistic properties and statistical methods.

[3] Bickel, P. J. and Wichura, M. J. (1971). Convergence criteria for multiparameter stochastic processes and some applications. Ann. Math. Statist., 42:1656-1670.

[4] Biermé, H. and Durieu, O. (2014). Invariance principles for self-similar set-indexed random fields. Trans. Amer. Math. Soc., 366(11):5963-5989.

[5] Biermé, H., Durieu, O., and Wang, Y. (2017). Invariance principles for operatorscaling Gaussian random fields. Ann. Appl. Probab., 27(2):1190-1234.

[6] Biermé, H., Meerschaert, M. M., and Scheffler, H.-P. (2007). Operator scaling stable random fields. Stochastic Process. Appl., 117(3):312-332.

[7] Biermé, H., Richard, F., Rachidi, M., and Benhamou, C.-L. (2009). Anisotropic texture modeling and applications to medical image analysis. In Mathematical methods for imaging and inverse problems, volume 26 of ESAIM Proc., pages 100-122. EDP Sci., Les Ulis.

[8] Durieu, O. and Wang, Y. (2016). From infinite urn schemes to decompositions of self-similar Gaussian processes. Electron. J. Probab., 21:Paper No. 43, 23.

[9] Durieu, O. and Wang, Y. (2019). From random partitions to fractional Brownian sheets. Bernoulli, 25(2):1412-1450.

[10] Enriquez, N. (2004). A simple construction of the fractional Brownian motion. Stochastic Process. Appl., 109(2):203-223.

[11] Granger, C. W. J. (1980). Long memory relationships and the aggregation of dynamic models. J. Econometrics, 14(2):227-238.

[12] Hammond, A. and Sheffield, S. (2013). Power law Pólya's urn and fractional Brownian motion. Probab. Theory Related Fields, 157(3-4):691-719.

[13] Kaj, I. and Taqqu, M. S. (2008). Convergence to fractional Brownian motion and to the Telecom process: the integral representation approach. In In and out of equilibrium. 2, volume 60 of Progr. Probab., pages 383-427. Birkhäuser, Basel.

[14] Lavancier, F. (2006). Long memory random fields. In Dependence in probability and statistics, volume 187 of Lecture Notes in Statist., pages 195-220. Springer, New York.

[15] Lavancier, F. (2007). Invariance principles for non-isotropic long memory random fields. Stat. Inference Stoch. Process., 10(3):255-282.

[16] Lavancier, F. (2011). Aggregation of isotropic autoregressive fields [corrigendum to mr2523650]. J. Statist. Plann. Inference, 141(12):3862-3866.

[17] Leonenko, N. and Taufer, E. (2013). Disaggregation of spatial autoregressive processes. Spatial Statistics, 3:1-20.

[18] Li, Y., Wang, W., and Xiao, Y. (2015). Exact moduli of continuity for operatorscaling Gaussian random fields. Bernoulli, 21(2):930-956.

[19] Lopes, R. and Betrouni, N. (2009). Fractal and multifractal analysis: a review. Medical image analysis, 13(4):634-649.

imsart-bj ver. 2014/10/16 file: aggregation20190726BEJ_combined.tex date: July 29, 2019 
Submitted to Bernoulli

arXiv: arXiv: 1712.08058

[20] Meerschaert, M. M., Dogan, M., Dam, R. L., Hyndman, D. W., and Benson, D. A. (2013a). Hydraulic conductivity fields: Gaussian or not? Water resources research, 49(8):4730-4737.

[21] Meerschaert, M. M., Wang, W., and Xiao, Y. (2013b). Fernique-type inequalities and moduli of continuity for anisotropic Gaussian random fields. Trans. Amer. Math. Soc., 365(2):1081-1107.

[22] Mikosch, T., Resnick, S., Rootzén, H., and Stegeman, A. (2002). Is network traffic approximated by stable Lévy motion or fractional Brownian motion? Ann. Appl. Probab., 12(1):23-68.

[23] Mikosch, T. and Samorodnitsky, G. (2007). Scaling limits for cumulative input processes. Math. Oper. Res., 32(4):890-918.

[24] Pilipauskaite, V. and Surgailis, D. (2017). Scaling transition for nonlinear random fields with long-range dependence. Stochastic Processes and their Applications, pages 2751-2779.

[25] Pinsky, M. A. (2002). Introduction to Fourier analysis and wavelets. Brooks/Cole Series in Advanced Mathematics. Brooks/Cole, Pacific Grove, CA.

[26] Pipiras, V. and Taqqu, M. S. (2017). Long-range dependence and self-similarity, volume 45 of Cambridge Series in Statistical and Probabilistic Mathematics. Cambridge University Press.

[27] Puplinskaite, D. and Surgailis, D. (2015). Scaling transition for long-range dependent Gaussian random fields. Stochastic Process. Appl., 125(6):2256-2271.

[28] Puplinskaite, D. and Surgailis, D. (2016). Aggregation of autoregressive random fields and anisotropic long-range dependence. Bernoulli, 22(4):2401-2441.

[29] Resnick, S. I. (2007). Heavy-tail phenomena. Springer Series in Operations Research and Financial Engineering. Springer, New York. Probabilistic and statistical modeling.

[30] Robinson, P. M. (1978). Statistical inference for a random coefficient autoregressive model. Scand. J. Statist., 5(3):163-168.

[31] Samorodnitsky, G. (2016). Stochastic processes and long range dependence. Springer, Cham, Switzerland.

[32] Samorodnitsky, G. and Taqqu, M. S. (1994). Stable non-Gaussian random processes. Stochastic Modeling. Chapman \& Hall, New York. Stochastic models with infinite variance.

[33] Shen, Y. and Wang, Y. (2017). Operator-scaling Gaussian random fields via aggregation. Submitted, available at https://arxiv.org/abs/1712.07082.

[34] Wang, Y. (2014). An invariance principle for fractional Brownian sheets. J. Theoret. Probab., 27(4):1124-1139.

[35] Xiao, Y. (2009). Sample path properties of anisotropic Gaussian random fields. In A minicourse on stochastic partial differential equations, volume 1962 of Lecture Notes in Math., pages 145-212. Springer, Berlin.

[36] Xiao, Y. (2013). Recent developments on fractal properties of Gaussian random fields. In Further developments in fractals and related fields, Trends Math., pages 255-288. Birkhäuser/Springer, New York. 


\title{
Supplement to "Operator-scaling Gaussian random fields via aggregation"
}

\author{
YI SHEN ${ }^{3}$ and YIZAO WANG ${ }^{4}$
}

${ }^{3}$ Department of Statistics and Actuarial Science, University of Waterloo, Mathematics 3

Building, 200 University Avenue West Waterloo, Ontario N2L 3G1, Canada.

E-mail: yi.shen@uwaterloo.ca

${ }^{4}$ Department of Mathematical Sciences, University of Cincinnati, 2815 Commons Way, ML-0025, Cincinnati, OH, 45221-0025, USA. E-mail: yizao.wang@uc.edu

We provide a proof of [33, Theorem 1.5] in Sections 5 and 6 below. The boundary case of the non-critical regime is investigated in Section 7.

\section{Proof of Theorem 1.4, regime (i)}

Recall that in this regime, we have

$$
\boldsymbol{n}^{\prime}=\boldsymbol{n}, n^{*}=n_{2}^{\alpha_{2}} \quad \text { with } \quad n_{1}^{\alpha_{1}} \gg n_{2}^{\alpha_{2}}, \alpha_{1}>1,
$$

which we assume throughout this section without further mentioning. Again we start by computing the second moments. We write

$$
\operatorname{Cov}\left(S_{\boldsymbol{n}}(\boldsymbol{s}), S_{\boldsymbol{n}}(\boldsymbol{t})\right)=\frac{|\boldsymbol{n}|^{-1}}{(2 \pi)^{2}} \int_{\boldsymbol{n} \cdot(-\pi, \pi)^{2}} D_{\boldsymbol{n}, \boldsymbol{s}, \boldsymbol{t}}(\boldsymbol{\theta} / \boldsymbol{n}) \widehat{r}(\boldsymbol{\theta} / \boldsymbol{n}) d \boldsymbol{\theta} .
$$

We have seen how to estimate and control $D$ in Lemma 3.3. We need the following estimates on $\widehat{r}(\boldsymbol{\theta} / \boldsymbol{n})$.

Proposition 5.1. Under assumption (5.1),

$$
\widehat{r}(\boldsymbol{\theta} / \boldsymbol{n}) \sim \frac{n_{2}^{2 H_{2}-1}}{\left|\theta_{2}\right|^{2 H_{2}-1}} \int_{\Delta_{1}} w_{1}^{1 / \alpha_{1}} w_{2}^{1-1 / \alpha_{1}} \Lambda(d \boldsymbol{w}) 2 \alpha_{2} B\left(H_{2}-\frac{1}{2}, \frac{3}{2}-H_{2}\right),
$$

and

$$
\widehat{r}(\boldsymbol{\theta} / \boldsymbol{n}) \leq C\left(\frac{n_{2}^{2 H_{2}-1}}{\left|\theta_{2}\right|^{2 H_{2}-1}}+1\right) .
$$

Proof. In this case, (4.2) becomes

$$
\widehat{r}(\boldsymbol{\theta} / \boldsymbol{n})=\left(n^{*}\right)^{-1} \int_{\Delta_{1}} \int_{0}^{\infty} \prod_{k=1}^{2} g\left(\left(n^{*} r w_{k}\right)^{-1 / \alpha_{k}}, \theta_{k} / n_{k}\right) \mathbf{1}_{\left\{n^{*} r \boldsymbol{w} \in T_{\boldsymbol{\alpha}}\left((0,1]^{2}\right)\right\}} \frac{d r}{r^{2}} \Lambda(d \boldsymbol{w}) .
$$


We start by proving the second part of the proposition. For the first direction (corresponding to $\left.H_{1}=1 / 2\right)$, we use the simple bound $g(u, \theta) \leq 2 u^{-1}$. For the second direction (corresponding to $H_{2} \in(1 / 2,1)$ ), again we break the integral into two parts. Introduce and fix $\delta \in(0,1)$. As in $(3.16)$, we have

$$
g\left(\left(n^{*} r w_{2}\right)^{-1 / \alpha_{2}}, \theta_{2} / n_{2}\right) \mathbf{1}_{\left\{\left(n^{*} r w_{2}\right)^{-1 / \alpha_{2}} \in(0, \delta]\right\}} \leq \frac{C\left(n^{*}\right)^{1 / \alpha_{2}}}{\left(r w_{2}\right)^{1 / \alpha_{2}} \theta_{2}^{2}}
$$

Then,

$$
\begin{aligned}
\int_{0}^{\infty} & \prod_{k=1}^{2} g\left(\left(n^{*} r w_{k}\right)^{-1 / \alpha_{k}}, \theta_{k} / n_{k}\right) \mathbf{1}_{\left\{\left(n^{*} r w_{2}\right)^{-1 / \alpha_{2}} \in(0, \delta]\right\}} \frac{d r}{r^{2}} \\
& \leq C\left(n^{*}\right)^{p(\boldsymbol{\alpha})} \int_{0}^{\infty} r^{1 / \alpha_{1}-2} w_{1}^{1 / \alpha_{1}} \min \left\{\left(r w_{2}\right)^{1 / \alpha_{2}}, \frac{1}{\left(r w_{2}\right)^{1 / \alpha_{2} \theta_{2}^{2}}}\right\} d r \\
& \leq C\left(n^{*}\right)^{p(\boldsymbol{\alpha})} \frac{w_{1}^{1 / \alpha_{1}} w_{2}^{1-1 / \alpha_{1}}}{\left|\theta_{2}\right|^{2 H_{2}-1}},
\end{aligned}
$$

where in the last step again we break the integral into two parts at $\left(r w_{2}\right)^{1 / \alpha_{2}}=1 /\left|\theta_{2}\right|$, and recall that in this case $\mathrm{H}_{2}$ is given in (4.12). At the same time,

$$
\begin{aligned}
\int_{0}^{\infty} \prod_{k=1}^{2} g & \left(\left(n^{*} r w_{k}\right)^{-1 / \alpha_{k}}, \theta_{k} / n_{k}\right) \mathbf{1}_{\left\{\left(n^{*} r w_{2}\right)^{-1 / \alpha_{2}} \in(\delta, 1]\right\}} \frac{d r}{r^{2}} \\
& =\int_{\left(n^{*} w_{2}\right)^{-1}}^{\left(n^{*} w_{2}\right)^{-1} \delta^{-\alpha_{2}}} \prod_{k=1}^{2} g\left(\left(n^{*} r w_{k}\right)^{-1 / \alpha_{k}}, \theta_{k} / n_{k}\right) \frac{d r}{r^{2}} \\
& \leq C\left(n^{*}\right)^{p(\boldsymbol{\alpha})} w_{1}^{1 / \alpha_{1}} w_{2}^{1 / \alpha_{2}} \int_{\left(n^{*} w_{2}\right)^{-1}}^{\left(n^{*} w_{2}\right)^{-1} \delta^{-\alpha_{2}}} r^{p(\boldsymbol{\alpha})-2} d r=C n^{*} w_{1}^{1 / \alpha_{1}} w_{2}^{1-1 / \alpha_{1}}
\end{aligned}
$$

where we used the bound $g(u, \theta) \leq 2 u^{-1}$ twice. Therefore, combining (5.2), (5.3) and (5.4), we have

$$
\widehat{r}(\boldsymbol{\theta} / \boldsymbol{n}) \leq \int_{\Delta_{1}} w_{1}^{1 / \alpha_{1}} w_{2}^{1-1 / \alpha_{1}} \Lambda(d \boldsymbol{w})\left(\left(n^{*}\right)^{p(\boldsymbol{\alpha})-1}\left|\theta_{2}\right|^{1-2 H_{2}}+1\right) .
$$

Remark that $\left(n^{*}\right)^{p(\boldsymbol{\alpha})-1}=n_{2}^{1+\alpha_{2} / \alpha_{1}-\alpha_{2}}=n_{2}^{2 H_{2}-1}$ by (4.12) and (5.1). This proves the second part of the proposition. For the first part, to show the asymptotics of (5.2), we have seen before

$$
\left(n^{*}\right)^{-p(\boldsymbol{\alpha})} \prod_{k=1}^{2} g\left(\left(n^{*} r w_{k}\right)^{-1 / \alpha_{k}}, \theta_{k} / n_{k}\right) \sim 2\left(r w_{1}\right)^{1 / \alpha_{1}} \frac{2\left(r w_{2}\right)^{-1 / \alpha_{2}}}{\left(r w_{2}\right)^{-2 / \alpha_{2}}+\theta_{2}^{2}} .
$$

So by the dominated convergence theorem and (4.10) (see (4.11)), the first part of the proposition follows. We omit the details.

imsart-bj ver. 2014/10/16 file: aggregation20190726BEJ_combined.tex date: July 29, 2019 
Proposition 5.2. Under assumption (5.1),

$$
\lim _{\boldsymbol{n} \rightarrow \infty} \frac{\operatorname{Cov}\left(S_{\boldsymbol{n}}(\boldsymbol{s}), S_{\boldsymbol{n}}(\boldsymbol{t})\right)}{n_{1}^{2 H_{1}} n_{2}^{2 H_{2}}}=\sigma^{2} \operatorname{Cov}\left(\mathbb{B}_{\boldsymbol{s}}^{\boldsymbol{H}}, \mathbb{B}_{\boldsymbol{t}}^{\boldsymbol{H}}\right),
$$

with $\boldsymbol{H}, \sigma$ as in regime (i) in Theorem 1.4.

Proof. By the estimates on $\widehat{r}(\boldsymbol{\theta} / \boldsymbol{n})$ and $D$ in Proposition 5.1 and Lemma 3.3 respectively, we have

$$
\begin{aligned}
\frac{\operatorname{Cov}\left(S_{\boldsymbol{n}}(\boldsymbol{s}), S_{\boldsymbol{n}}(\boldsymbol{t})\right)}{n_{1}^{2 H_{1}} n_{2}^{2 H_{2}}}= & \frac{|\boldsymbol{n}|^{-1}}{n_{1} n_{2}^{2 H_{2}}(2 \pi)^{2}} \int_{\boldsymbol{n} \cdot(-\pi, \pi)^{2}} D_{\boldsymbol{n}, \boldsymbol{s}, \boldsymbol{t}}(\boldsymbol{\theta} / \boldsymbol{n}) \widehat{r}(\boldsymbol{\theta} / \boldsymbol{n}) d \boldsymbol{\theta} \\
\sim & \frac{1}{(2 \pi)^{2}} \int_{\mathbb{R}^{2}} \prod_{k=1}^{2} \frac{\left(e^{i s_{k} \theta_{k}}-1\right) \overline{\left(e^{i t_{k} \theta_{k}}-1\right)}}{\left|\theta_{k}\right|^{2}} \frac{1}{\left|\theta_{2}\right|^{2 H_{2}-1}} d \boldsymbol{\theta} \\
& \times \int_{\Delta_{1}} w_{1}^{1 / \alpha_{1}} w_{2}^{1-1 / \alpha_{1}} \Lambda(d \boldsymbol{w}) 2 \alpha_{2} B\left(H_{2}-\frac{1}{2}, \frac{3}{2}-H_{2}\right) \\
= & \sigma^{2} \operatorname{Cov}\left(\mathbb{B}_{\boldsymbol{s}}^{\boldsymbol{H}}, \mathbb{B}_{\boldsymbol{t}}^{\boldsymbol{H}}\right),
\end{aligned}
$$

by the dominated convergence theorem.

Proposition 5.3. For $\boldsymbol{t}$ such that $\lfloor\boldsymbol{n} \cdot \boldsymbol{t}\rfloor=\boldsymbol{n} \cdot \boldsymbol{t}$, we have

$$
\mathbb{E} S_{\boldsymbol{n}}(\boldsymbol{t})^{4} \leq C n_{1}^{3} n_{2}^{2 H_{2}+2} t_{1}^{3} t_{2}^{2 H_{2}+2} .
$$

Proof. By (3.21) and (3.22) and Proposition 5.1, we have

$$
\begin{aligned}
\mathbb{E} S_{\boldsymbol{n}}(\boldsymbol{t})^{4} & \leq C|\boldsymbol{n}|^{-1} \int_{\boldsymbol{n} \cdot(-\pi, \pi)^{2}} J_{\lfloor\boldsymbol{n} \cdot \boldsymbol{t}\rfloor}^{*}(\boldsymbol{\theta} / \boldsymbol{n}) \widehat{r}(\boldsymbol{\theta} / \boldsymbol{n}) d \boldsymbol{\theta} \\
& \leq C|\boldsymbol{n}|^{-1}|\boldsymbol{n}|^{4}|\boldsymbol{t}|^{2} \int_{\mathbb{R}^{2}} \prod_{k=1}^{2} \min \left\{t_{k}^{2}, \frac{1}{\left|\theta_{k}\right|^{2}}\right\}\left(n_{2}^{2 H_{2}-1}\left|\theta_{2}\right|^{1-2 H_{2}}+1\right) d \boldsymbol{\theta} \\
& \leq C\left(n_{1}^{3} n_{2}^{2 H_{2}+2} t_{1}^{3} t_{2}^{2 H_{2}+2}+|\boldsymbol{n}|^{3}|\boldsymbol{t}|^{3}\right) .
\end{aligned}
$$

The desired result now follows, by noticing that $H_{2} \in(1 / 2,1)$.

Proof of Theorem 1.4, regime (i). Set $Y_{\boldsymbol{n}}$ as in (2.5). We only verify the LindebergFeller condition. This time we have

$$
\begin{aligned}
\mathbb{E}\left(Y_{\boldsymbol{n}}^{2} \mathbf{1}_{\left\{Y_{\boldsymbol{n}}^{2}>m(\boldsymbol{n}) \eta\right\}}\right) & \leq \frac{1}{m(\boldsymbol{n}) \eta} \mathbb{E} Y_{n}^{4} \leq \frac{1}{m(\boldsymbol{n}) \eta}\left(\frac{1}{n_{1}^{1 / 2} n_{2}^{H_{2}}} \sum_{w=1}^{d}\left|a_{w}\right|\left(\mathbb{E} S_{\boldsymbol{n}}^{4}\left(\boldsymbol{t}_{w}\right)\right)^{1 / 4}\right)^{4} \\
& \leq \frac{C}{m(\boldsymbol{n}) \eta n_{1}^{2} n_{2}^{4 H_{2}}} n_{1}^{3} n_{2}^{2 H_{2}+2}=\frac{C}{\eta} \frac{n_{1} n_{2}^{2-2 H_{2}}}{m(\boldsymbol{n})}
\end{aligned}
$$

This completes the proof. 


\section{Proof of Theorem 1.4, regime (ii)}

Recall that in this regime,

$$
n^{*}=n_{1}^{\alpha_{1}} \quad \text { and } \quad \boldsymbol{n}^{\prime}=\left(n_{1}, n_{1}^{\alpha_{1} / \alpha_{2}}\right) \quad \text { with } \quad \alpha_{1}<1, n_{1}^{\alpha_{1}} \gg n_{2}^{\alpha_{2}},
$$

which we assume throughout without further specification. The treatment is slightly different from the previous cases in the sense that we have to work with the representation of covariance function as a triple integral (see (6.2) below), and apply the dominated convergence theorem once for all.

Proposition 6.1. Under the assumption (6.1), we have

$$
\lim _{\boldsymbol{n} \rightarrow \infty} \frac{\operatorname{Cov}\left(S_{\boldsymbol{n}}(\boldsymbol{s}), S_{\boldsymbol{n}}(\boldsymbol{t})\right)}{n_{1}^{2 H_{1}} n_{2}^{2 H_{2}}}=\sigma^{2} \operatorname{Cov}\left(\mathbb{B}_{\boldsymbol{s}}^{\boldsymbol{H}}, \mathbb{B}_{\boldsymbol{t}}^{\boldsymbol{H}}\right),
$$

with $\boldsymbol{H}=\left(1-\alpha_{1} / 2,1\right)$, and $\sigma$ as in regime (ii) in Theorem 1.4.

Proof. This time, to simplify the notation we introduce

$$
\widetilde{h}_{\boldsymbol{n}}(r, \boldsymbol{w}, \boldsymbol{\theta}):=\prod_{k=1}^{2} g\left(\left(n^{*} r w_{k}\right)^{-1 / \alpha_{k}}, \theta_{k} / n_{k}^{\prime}\right) \mathbf{1}_{\left\{n^{*} r \boldsymbol{w} \in T_{\boldsymbol{\alpha}}\left((0,1]^{2}\right)\right\}} .
$$

Combining (4.1) and (4.2), we have

$$
\begin{aligned}
& \frac{\operatorname{Cov}\left(S_{\boldsymbol{n}}(\boldsymbol{s}), S_{\boldsymbol{n}}(\boldsymbol{t})\right)}{n_{1}^{2 H_{1}} n_{2}^{2 H_{2}}} \\
& =\frac{1}{\left|\boldsymbol{n}^{\prime}\right||\boldsymbol{n}|^{2}(2 \pi)^{2}} \int_{\Delta_{1}} \int_{0}^{\infty} \int_{\boldsymbol{n}^{\prime} \cdot(-\pi, \pi)^{2}} D_{\boldsymbol{n}, \boldsymbol{s}, \boldsymbol{t}}\left(\boldsymbol{\theta} / \boldsymbol{n}^{\prime}\right) \widetilde{h}_{\boldsymbol{n}}(r, \boldsymbol{w}, \boldsymbol{\theta}) r^{-2} d \boldsymbol{\theta} d r \Lambda(d \boldsymbol{w}) .
\end{aligned}
$$

We shall apply the dominated convergence theorem. We have seen the pointwise convergence of the normalized integrand in Section 4, so it suffices to find an integrable bound. Similarly as in the critical case, we divide this integral into two parts with domains determined by

$$
\Omega_{n}^{0,0}:=\left\{n^{*} r \boldsymbol{w} \in T_{\boldsymbol{\alpha}}\left((0, \delta]^{2}\right)\right\} \quad \text { and } \quad \Omega_{\boldsymbol{n}}:=\left\{n^{*} r \boldsymbol{w} \in T_{\boldsymbol{\alpha}}\left((0,1]^{2} \backslash(0, \delta]^{2}\right)\right\},
$$

respectively. Introduce also $\Xi_{\boldsymbol{n}^{\prime}}:=\left\{\boldsymbol{\theta} \in \boldsymbol{n}^{\prime} \cdot(-\pi, \pi)^{2}\right\}$. We first justify the application of the dominated convergence theorem to

$$
\frac{1}{\left|\boldsymbol{n}^{\prime}\right||\boldsymbol{n}|^{2}(2 \pi)^{2}} \int_{\Delta_{1}} \int_{0}^{\infty} \int_{\mathbb{R}^{2}} D_{\boldsymbol{n}, \boldsymbol{s}, \boldsymbol{t}}\left(\boldsymbol{\theta} / \boldsymbol{n}^{\prime}\right) \mathbf{1}_{\Xi_{n^{\prime}}} \widetilde{h}_{\boldsymbol{n}}(r, \boldsymbol{w}, \boldsymbol{\theta}) \mathbf{1}_{\Omega_{\boldsymbol{n}}^{0,0}} r^{-2} d \boldsymbol{\theta} d r \Lambda(d \boldsymbol{w}) .
$$

Similarly as in Lemma 3.3, we have, for $k=1,2,\left|\theta_{k}\right| \leq n_{k}^{\prime} \pi$,

$$
\left|\frac{D_{\left\lfloor n_{k} t_{k}\right\rfloor}\left(\theta_{k} / n_{k}^{\prime}\right)}{n_{k}}\right|=\frac{\left|\sin \left(\left\lfloor n_{k} t_{k}\right\rfloor \theta_{k} / 2 n_{k}^{\prime}\right)\right|}{n_{k}\left|\sin \left(\theta_{k} / 2 n_{k}^{\prime}\right)\right|} \leq \min \left\{\frac{\pi t_{k}}{2}, \frac{n_{k}^{\prime}}{\left|\theta_{k}\right| n_{k}}\right\} .
$$

imsart-bj ver. 2014/10/16 file: aggregation20190726BEJ_combined.tex date: July 29, 2019 
Hence

$$
\left|D_{\boldsymbol{n}, \boldsymbol{s}, \boldsymbol{t}}\left(\boldsymbol{\theta} / \boldsymbol{n}^{\prime}\right)\right| \mathbf{1}_{\Xi_{\boldsymbol{n}^{\prime}}} \leq\left(\frac{\pi}{2}\right)^{4}|\boldsymbol{n}|^{2} \min \left\{s_{1} t_{1}, \frac{1}{\left|\theta_{1}\right|^{2}}\right\} s_{2} t_{2} .
$$

On $\Omega_{n}^{0,0}$, using in addition

$$
\begin{aligned}
g\left(\left(n^{*} r w_{k}\right)^{-1 / \alpha_{k}}, \theta_{k} / n_{k}^{\prime}\right) & \leq \frac{2\left(n^{*} r w_{k}\right)^{1 / \alpha_{k}}}{1+C_{\delta}\left(n^{*} r w_{k}\right)^{2 / \alpha_{k}}\left(1-\cos \left(\theta_{k} /\left(n^{*}\right)^{1 / \alpha_{k}}\right)\right.} \\
& \leq \frac{2\left(n^{*} r w_{k}\right)^{1 / \alpha_{k}}}{1+C_{\delta} \theta_{k}^{2}\left(r w_{k}\right)^{2 / \alpha_{k}}},
\end{aligned}
$$

where we used the fact that $1-\cos \theta \geq 2 \theta^{2} / \pi^{2}$ for all $\theta \in[-\pi, \pi]$, we bound the integrand of (6.3) (with the normalization $\left(\left|\boldsymbol{n}^{\prime}\right||\boldsymbol{n}|^{2}\right)^{-1}$ ) by

$$
C \min \left\{s_{1} t_{1}, \frac{1}{\theta_{1}^{2}}\right\} s_{2} t_{2} \prod_{k=1}^{2} \frac{\left(r w_{k}\right)^{1 / \alpha_{k}}}{1+C_{\delta}\left(r w_{k}\right)^{2 / \alpha_{k}} \theta_{k}^{2}} r^{-2} .
$$

To see that this is integrable, we integrate it with respect to $d \theta_{2}, d r$ and $d \theta_{1}$ in order and obtain

$$
\begin{aligned}
& \int_{\Delta_{1}} \int_{\mathbb{R}^{2}} \int_{\mathbb{R}_{+}} \min \left\{s_{1} t_{1}, \frac{1}{\theta_{1}^{2}}\right\} s_{2} t_{2} \prod_{k=1}^{2} \frac{\left(r w_{k}\right)^{1 / \alpha_{k}}}{1+C_{\delta}\left(r w_{k}\right)^{2 / \alpha_{k}} \theta_{k}^{2}} r^{-2} d r d \boldsymbol{\theta} \Lambda(d \boldsymbol{w}) \\
& =C \int_{\Delta_{1}} \int_{\mathbb{R}} \int_{\mathbb{R}_{+}} \min \left\{s_{1} t_{1}, \frac{1}{\theta_{1}^{2}}\right\} s_{2} t_{2} \frac{\left(r w_{1}\right)^{1 / \alpha_{1}}}{1+C_{\delta}\left(r w_{1}\right)^{2 / \alpha_{1}} \theta_{1}^{2}} \frac{d r}{r^{2}} d \theta_{1} \Lambda(d \boldsymbol{w}) \\
& =C_{\delta} \int_{\Delta_{1}} \int_{\mathbb{R}} \min \left\{s_{1} t_{1}, \frac{1}{\theta_{1}^{2}}\right\} s_{2} t_{2} w_{1}\left|\theta_{1}\right|^{\alpha_{1}-1} d \theta_{1} \Lambda(d \boldsymbol{w}) \\
& =C_{\delta}\left(s_{1} t_{1}\right)^{1-\alpha_{1} / 2} s_{2} t_{2} \int_{\Delta_{1}} w_{1} \Lambda(d \boldsymbol{w}) \leq C_{\delta}\left(s_{1} t_{1}\right)^{1-\alpha_{1} / 2} s_{2} t_{2} .
\end{aligned}
$$

Next, we show that (6.3) with $\Omega_{n}^{0,0}$ replacedy by $\Omega_{\boldsymbol{n}}$ converges to zero for any given $\delta \in(0,1)$. Again we divide the region of interest into three pieces, $\Omega_{\boldsymbol{n}}=\Omega_{\boldsymbol{n}}^{0,1} \cup \Omega_{\boldsymbol{n}}^{1,0} \cup \Omega_{\boldsymbol{n}}^{1,1}$ with

$$
\begin{aligned}
& \Omega_{\boldsymbol{n}}^{0,1}:=\left\{n^{*} r \boldsymbol{w} \in T_{\boldsymbol{\alpha}}((0, \delta] \times(\delta, 1])\right\} \\
& \Omega_{\boldsymbol{n}}^{1,0}:=\left\{n^{*} r \boldsymbol{w} \in T_{\boldsymbol{\alpha}}((\delta, 1] \times(0, \delta])\right\} \\
& \Omega_{\boldsymbol{n}}^{1,1}:=\left\{n^{*} r \boldsymbol{w} \in T_{\boldsymbol{\alpha}}\left((\delta, 1]^{2}\right)\right\},
\end{aligned}
$$

and on each we apply the inequality

$$
\begin{aligned}
& g\left(\left(n^{*} r w_{k}\right)^{-1 / \alpha_{k}}, \theta_{k} / n_{k}^{\prime}\right) \\
& \quad \leq \begin{cases}C_{\delta} n_{k}^{\prime} \min \left\{\left(r w_{k}\right)^{1 / \alpha_{k}}, \frac{1}{\left(r w_{k}\right)^{1 / \alpha_{k}} \theta_{k}^{2}}\right\} & \left(n^{*} r w_{k}\right)^{-1 / \alpha_{k}} \in(0, \delta] \\
C_{\delta} & \left(n^{*} r w_{k}\right)^{-1 / \alpha_{k}} \in(\delta, 1]\end{cases}
\end{aligned}
$$


For $\Omega_{n}^{1,1}$, by (6.4) and the above inequality, the integral (6.3) with $\Omega_{n}^{0,0}$ replaced by $\Omega_{n}^{1,1}$ is bounded by

$$
\begin{gathered}
\frac{C_{\delta} n_{2}^{\prime}|\boldsymbol{n}|^{2} s_{2} t_{2}}{\left|\boldsymbol{n}^{\prime}\right||\boldsymbol{n}|^{2}} \int_{\mathbb{R}} \min \left\{s_{1} t_{1}, \frac{1}{\left|\theta_{1}\right|^{2}}\right\} d \theta_{1} \int_{\Delta_{1}} \int_{\left(n^{*}\right)^{-1}\left(w_{1}^{-1} \vee w_{2}^{-1}\right)}^{\left(n^{*}\right)^{-1}\left[\left(w_{1} \delta^{\alpha_{1}}\right)^{-1} \vee\left(w_{2} \delta^{\alpha_{2}}\right)^{-1}\right]} r^{-2} d r \Lambda(d \boldsymbol{w}) \\
\leq \frac{C_{\delta} n_{2}^{\prime} n^{*}}{\left|\boldsymbol{n}^{\prime}\right|}\left(s_{1} t_{1}\right)^{1 / 2} s_{2} t_{2} \int_{\Delta_{1}} w_{1} \wedge w_{2} \Lambda(d \boldsymbol{w}) \leq C_{\delta}\left(s_{1} t_{1}\right)^{1 / 2} s_{2} t_{2} n_{1}^{\alpha_{1}-1} \rightarrow 0 .
\end{gathered}
$$

Similarly, (6.3) with $\Omega_{n}^{0,0}$ replaced by $\Omega_{n}^{1,0}$ is bounded by

$$
\begin{aligned}
\frac{C_{\delta} n_{2}^{\prime} s_{2} t_{2}}{\left|\boldsymbol{n}^{\prime}\right|} \int_{\Delta_{1}} \int_{\left(n^{*} w_{1}\right)^{-1}}^{\infty} \int_{\mathbb{R}} \min \left\{s_{1} t_{1}, \frac{1}{\left|\theta_{1}\right|^{2}}\right\} d \theta_{1} \\
\qquad \int_{\mathbb{R}} \min \left\{\left(r w_{2}\right)^{1 / \alpha_{2}}, \frac{1}{\left(r w_{2}\right)^{1 / \alpha_{2}} \theta_{2}^{2}}\right\} d \theta_{2} r^{-2} d r \Lambda(d \boldsymbol{w}),
\end{aligned}
$$

which then becomes

$$
\begin{aligned}
\frac{C_{\delta}}{n_{1}^{\prime}}\left(s_{1} t_{1}\right)^{1 / 2} s_{2} t_{2} & \int_{\Delta_{1}} \int_{\left(n^{*} w_{1}\right)^{-1}}^{\infty} r^{-2} d r \Lambda(d \boldsymbol{w}) \\
& \leq \frac{C_{\delta} n^{*}}{n_{1}^{\prime}}\left(s_{1} t_{1}\right)^{1 / 2} s_{2} t_{2} \int_{\Delta_{1}} w_{1} \Lambda(d \boldsymbol{w}) \leq C_{\delta}\left(s_{1} t_{1}\right)^{1 / 2} s_{2} t_{2} n_{1}^{\alpha_{1}-1} \rightarrow 0 .
\end{aligned}
$$

The last area $\Omega_{n}^{0,1}$ requires a more careful treatment. In this case, (6.3) with $\Omega_{n}^{0,0}$ replaced by $\Omega_{n}^{0,1}$ is bounded by

$$
C_{\delta} \int_{\mathbb{R}} \int_{\Delta_{1}} \int_{\mathbb{R}} \min \left\{s_{1} t_{1}, \frac{1}{\left|\theta_{1}\right|^{2}}\right\} s_{2} t_{2} \frac{1}{n_{1}^{\prime}} g\left(\left(n^{*} r w_{1}\right)^{-1 / \alpha_{1}}, \theta_{1} / n_{1}^{\prime}\right) \mathbf{1}_{\Omega_{n}^{0,1}} r^{-2} d r \Lambda(d \boldsymbol{w}) d \theta_{1} .
$$

Note that the current integrand converges pointwisely to 0 as $n^{*}$ goes to infinity, since $n^{*} r \boldsymbol{w}$ will eventually leave the area $T_{\boldsymbol{\alpha}}((0, \delta] \times(\delta, 1])$. Hence in order to prove the integral converges to 0 as well, it suffices to find an integrable upper bound, and then to apply the dominated convergence theorem. This can be done by applying bound (6.6) again:

$$
\begin{aligned}
\min \left\{s_{1} t_{1}, \frac{1}{\left|\theta_{1}\right|^{2}}\right\} s_{2} t_{2} \frac{1}{n_{1}^{\prime}} g\left(\left(n^{*} r w_{1}\right)^{-1 / \alpha_{1}}, \theta_{1} / n_{1}^{\prime}\right) \mathbf{1}_{\Omega_{n}^{0,1}} r^{-2} \\
\leq C_{\delta} s_{2} t_{2} \min \left\{s_{1} t_{1}, \frac{1}{\left|\theta_{1}\right|^{2}}\right\} \min \left\{\left(r w_{1}\right)^{1 / \alpha_{1}}, \frac{1}{\left(r w_{1}\right)^{1 / \alpha_{1}} \theta_{1}^{2}}\right\} r^{-2},
\end{aligned}
$$

which is integrable:

$$
\begin{aligned}
& \int_{\mathbb{R}} \int_{\Delta_{1}} \int_{\mathbb{R}} C_{\delta} s_{2} t_{2} \min \left\{s_{1} t_{1}, \frac{1}{\left|\theta_{1}\right|^{2}}\right\} \min \left\{\left(r w_{1}\right)^{1 / \alpha_{1}}, \frac{1}{\left(r w_{1}\right)^{1 / \alpha_{1}} \theta_{1}^{2}}\right\} r^{-2} d r \Lambda(d \boldsymbol{w}) d \theta_{1} \\
& =C_{\delta} s_{2} t_{2} \int_{\mathbb{R}} \min \left\{s_{1} t_{1}, \frac{1}{\left|\theta_{1}\right|^{2}}\right\} \int_{\Delta_{1}} \theta_{1}^{\alpha_{1}-1} w_{1} \Lambda(d \boldsymbol{w}) d \theta_{1} \\
& \leq C_{\delta} s_{2} t_{2} \int_{\mathbb{R}} \min \left\{s_{1} t_{1}, \frac{1}{\left|\theta_{1}\right|^{2}}\right\} \theta_{1}^{\alpha_{1}-1} d \theta_{1}=C_{\delta} s_{1}^{1-\alpha_{1} / 2} t_{1}^{1-\alpha_{1} / 2} s_{2} t_{2}
\end{aligned}
$$


Therefore, we conclude that (6.3) with $\Omega_{n}^{0,0}$ replaced by $\Omega_{n}$ vanishes asymptotically. This completes the proof.

It remains to bound the fourth moment.

Proposition 6.2. Under the assumption (6.1), for $\boldsymbol{t}$ such that $\lfloor\boldsymbol{n} \cdot \boldsymbol{t}\rfloor=\boldsymbol{n} \cdot \boldsymbol{t}$, we have

$$
\mathbb{E} S_{\boldsymbol{n}}(\boldsymbol{t})^{4} \leq C n_{1}^{2 H_{1}+2} n_{2}^{4} t_{1}^{2 H_{1}+2} t_{2}^{4} .
$$

Proof.

$$
\begin{aligned}
\mathbb{E} S_{\boldsymbol{n}}(\boldsymbol{t})^{4} & \leq C\left|\boldsymbol{n}^{\prime}\right|^{-1} \int_{\mathbb{R}^{2}} J_{\lfloor\boldsymbol{n} \cdot \boldsymbol{t}\rfloor}^{*}\left(\boldsymbol{\theta} / \boldsymbol{n}^{\prime}\right) \mathbf{1}_{\Xi_{n^{\prime}}} \widehat{r}\left(\boldsymbol{\theta} / \boldsymbol{n}^{\prime}\right) d \boldsymbol{\theta} \\
& =C \frac{1}{\left|\boldsymbol{n}^{\prime}\right| n^{*}} \int_{\Delta_{1}} \int_{0}^{\infty} \int_{\mathbb{R}^{2}} J_{\lfloor\boldsymbol{n} \cdot \boldsymbol{t}\rfloor}^{*}\left(\boldsymbol{\theta} / \boldsymbol{n}^{\prime}\right) \mathbf{1}_{\Xi_{n^{\prime}}} \widetilde{h}_{\boldsymbol{n}}(r, \boldsymbol{w}, \boldsymbol{\theta}) r^{-2} d \boldsymbol{\theta} d r \Lambda(d \boldsymbol{w}) .
\end{aligned}
$$

The upper bound of $J$ in (3.24) (recall that $n_{2}^{\prime}=n_{1}^{\alpha_{1} / \alpha_{2}} \gg n_{2}$ ) this time yields

$$
J_{\lfloor\boldsymbol{n} \cdot \boldsymbol{t}\rfloor}^{*}\left(\boldsymbol{\theta} / \boldsymbol{n}^{\prime}\right) \mathbf{1}_{\Xi_{n^{\prime}}} \leq C|\boldsymbol{n}|^{4} t_{1}^{2} t_{2}^{4} \min \left\{t_{1}^{2}, \frac{1}{\theta_{1}^{2}}\right\} .
$$

Therefore, the integrand of (6.10) is bounded by

$$
|\boldsymbol{n}|^{2}\left(t_{1} t_{2}\right)^{2} \cdot|\boldsymbol{n}|^{2} \min \left\{t_{1}^{2}, \frac{1}{\theta_{1}^{2}}\right\} t_{2}^{2} \widetilde{h}_{\boldsymbol{n}}(r, \boldsymbol{w}, \boldsymbol{\theta}) r^{-2} .
$$

In view of (6.4), we recognize that one can obtain an upper bound for the integral in (6.10) that differs from the upper bound for the integral in (6.3) in the previous case by a multiplicative constant $C_{\delta}|\boldsymbol{n}|^{2}\left(t_{1} t_{2}\right)^{2}$. In particular, combining (6.5), (6.7), (6.8) and (6.9), we thus arrive at

$$
\begin{aligned}
\mathbb{E} S_{\boldsymbol{n}}(\boldsymbol{t})^{4} & \leq C_{\delta} \frac{1}{\left|\boldsymbol{n}^{\prime}\right| n^{*}} \cdot|\boldsymbol{n}|^{2}\left(t_{1} t_{2}\right)^{2} \cdot\left|\boldsymbol{n}^{\prime}\right||\boldsymbol{n}|^{2}\left(t_{1} t_{2}^{2} n_{1}^{\alpha_{1}-1}+t_{1}^{2-\alpha_{1}} t_{2}^{2}\right) \\
& \leq C_{\delta} n_{1}^{4-\alpha_{1}} n_{2}^{4}\left(t_{1} t_{2}\right)^{2}\left(t_{1} t_{2}^{2} n_{1}^{\alpha_{1}-1}+t_{1}^{2-\alpha_{1}} t_{2}^{2}\right) \\
& =C_{\delta}\left[\left(n_{1} t_{1}\right)^{4-\alpha_{1}}\left(n_{2} t_{2}\right)^{4}+\left(n_{1} t_{1}\right)^{3}\left(n_{2} t_{2}\right)^{4}\right] .
\end{aligned}
$$

By our assumption, $\left(n_{1} t_{1}\right)^{3} \leq\left(n_{1} t_{1}\right)^{4-\alpha_{1}}$, hence the desired result follows.

\section{Boundary case of non-critical speed}

The case for non-critical speed

$$
\alpha_{1}=1 \quad \text { and } \quad n_{1} \gg n_{2}^{\alpha_{2}},
$$


was not discussed in Theorem 1.4. As one would expect by observing the two regimes in Theorem 1.4, the case with $\alpha_{1}=1$ is degenerate and the limit is a fractional Brownian sheet with Hurst indices

$$
\boldsymbol{H}=(1 / 2,1) \text {. }
$$

The normalization in the functional limit theorem involves this time, however, a logarithmic term in $\boldsymbol{n}$. We expect to be able to show that

$$
\frac{\widehat{S}_{\boldsymbol{n}}(\boldsymbol{t})}{\sqrt{n_{1} \log n_{1}} n_{2} \sqrt{m(\boldsymbol{n})}} \Rightarrow \sigma\left\{\mathbb{B}_{\boldsymbol{t}}^{\boldsymbol{H}}\right\}_{\boldsymbol{t} \in[0,1]^{2}},
$$

with

$$
\sigma^{2}=4 \pi \int_{\Delta_{1}} w_{1} \Lambda(d \boldsymbol{w})
$$

Here, we only prove the limit theorem for the covariance function of a single random field.

Proposition 7.1. With (7.1), if

$$
\int_{\Delta_{1}}\left|\log w_{2}\right| \Lambda(d \boldsymbol{w})<\infty
$$

then,

$$
\lim _{\boldsymbol{n} \rightarrow \infty} \frac{\operatorname{Cov}\left(S_{\boldsymbol{n}}(\boldsymbol{s}), S_{\boldsymbol{n}}(\boldsymbol{t})\right)}{n_{1} \log \left(n_{1}\right) n_{2}^{2}}=\sigma^{2} \operatorname{Cov}\left(\mathbb{B}_{\boldsymbol{s}}^{\boldsymbol{H}}, \mathbb{B}_{\boldsymbol{t}}^{\boldsymbol{H}}\right)
$$

Proof. Under the assumption (7.1), we take

$$
\boldsymbol{n}^{\prime}=\left(n_{1}, n_{1}^{1 / \alpha_{2}}\right), n^{*}=n_{1} .
$$

We still need to work with triple integral representation of the covariance function

$$
\begin{aligned}
\frac{\operatorname{Cov}\left(S_{\boldsymbol{n}}(\boldsymbol{s}), S_{\boldsymbol{n}}(\boldsymbol{t})\right)}{n_{1} \log \left(n_{1}\right) n_{2}^{2}}= & \frac{1}{(2 \pi)^{2} \log n_{1}} \int_{\Delta_{1}} \int_{0}^{\infty} \int_{\boldsymbol{n}^{\prime} \cdot(-\pi, \pi)^{2}} \mathbf{1}_{\left\{n^{*} r \boldsymbol{w} \in T_{\boldsymbol{\alpha}}\left((0,1]^{2}\right)\right\}} \\
& \times \frac{D_{\boldsymbol{n}, \boldsymbol{s}, \boldsymbol{t}}\left(\boldsymbol{\theta} / \boldsymbol{n}^{\prime}\right)}{|\boldsymbol{n}|^{2}} \prod_{k=1}^{2} \frac{g\left(\left(n^{*} r w_{k}\right)^{-1 / \alpha_{k}}, \theta_{k} / n_{k}^{\prime}\right)}{\left(n^{*}\right)^{1 / \alpha_{k}}} d \boldsymbol{\theta} \frac{d r}{r^{2}} \Lambda(d \boldsymbol{w}) .
\end{aligned}
$$

With our choice of $\boldsymbol{n}^{\prime}$, we have that formally, by (4.3) and (4.4), (7.3) is asymptotically equivalent to

$$
\begin{aligned}
& \frac{s_{2} t_{2}}{(2 \pi)^{2} \log n_{1}} \\
& \quad \times \int_{\Delta_{1}} \int_{1 /\left(n^{*}\left(w_{1} \wedge w_{2}\right)\right)}^{\infty} \int_{\mathbb{R}^{2}} \frac{\left(e^{i s_{1} \theta_{1}}-1\right) \overline{\left(e^{i t_{1} \theta_{1}}-1\right)}}{\left|\theta_{1}\right|^{2}} \prod_{k=1}^{2} \frac{2\left(r w_{k}\right)^{-1 / \alpha_{k}}}{\left(r w_{k}\right)^{-2 / \alpha_{k}}+\theta_{k}^{2}} d \boldsymbol{\theta} \frac{d r}{r^{2}} \Lambda(d \boldsymbol{w}) . \\
& \text { imsart-bj ver. 2014/10/16 file: aggregation20190726BEJ_combined.tex date: July 29, } 2019
\end{aligned}
$$


For the above triple integral, we first integrate with respect to $d \theta_{2}$. The above then becomes

$$
\frac{s_{2} t_{2}}{2 \pi \log n_{1}} \int_{\Delta_{1}} \int_{\mathbb{R}} \frac{\left(e^{i s_{1} \theta_{1}}-1\right) \overline{\left(e^{i t_{1} \theta_{1}}-1\right)}}{\left|\theta_{1}\right|^{2}} \int_{1 /\left(n^{*}\left(w_{1} \wedge w_{2}\right)\right)}^{\infty} \frac{2\left(r w_{1}\right)^{-1}}{\left(r w_{1}\right)^{-2}+\theta_{1}^{2}} \frac{d r}{r^{2}} d \theta_{1} \Lambda(d \boldsymbol{w}) .
$$

Now, for the inner integral with respect to $d r$, we write it as

$$
\begin{aligned}
\int_{0}^{n^{*}\left(w_{1} \wedge w_{2}\right)} \frac{2 r w_{1}^{-1}}{r^{2} w_{1}^{-2}+\theta_{1}^{2}} d r & =w_{1} \int_{0}^{n^{*}\left(w_{1} \wedge w_{2}\right) /\left(w_{1} \theta_{1}\right)} \frac{2 r}{r^{2}+1} d r \\
& =w_{1} \log \left[1+\left(n^{*} \frac{w_{1} \wedge w_{2}}{w_{1} \theta_{1}}\right)^{2}\right] \sim 2 w_{1} \log n^{*} .
\end{aligned}
$$

To sum up, we have formally shown that

$$
\begin{aligned}
\frac{\operatorname{Cov}\left(S_{\boldsymbol{n}}(\boldsymbol{s}), S_{\boldsymbol{n}}(\boldsymbol{t})\right)}{n_{1} \log \left(n_{1}\right) n_{2}^{2}} & =\frac{s_{2} t_{2}}{\pi} \int_{\Delta_{1}} \int_{-\infty}^{\infty} \frac{\left(e^{i s_{1} \theta_{1}}-1\right) \overline{\left(e^{i t_{1} \theta_{1}}-1\right)}}{\left|\theta_{1}\right|^{2}} d \theta_{1} w_{1} \Lambda(d \boldsymbol{w}) \\
& =\operatorname{Cov}\left(\mathbb{B}_{\boldsymbol{s}}^{\boldsymbol{H}}, \mathbb{B}_{\boldsymbol{t}}^{\boldsymbol{H}}\right) \cdot 4 \pi \int_{\Delta_{1}} w_{1} \Lambda(d \boldsymbol{w}),
\end{aligned}
$$

where $\boldsymbol{H}=(1 / 2,1)$. Now we provide a rigorous proof of this convergence. We shall apply several approximations in a row. Again we start with (7.3). Recall our notations for $\Omega_{n}^{0,0}$ and $\Omega_{n}$. We first remark that for this integration over $\Omega_{n}$ and without the $\log n_{1}$ normalization, it is same as (6.3) with $\Omega_{n}^{0,0}$ replaced by $\Omega_{\boldsymbol{n}}$, and can be bounded by $C_{\delta}$ (see (6.7), (6.8) and (6.9)). Therefore, the contribution to the limit comes from the integration over $\Omega_{n}^{0,0}$.

Set

$$
D_{n, s, t}(\theta)=\operatorname{Re}\left(D_{\lfloor n s\rfloor}(\theta) \overline{D_{\lfloor n t\rfloor}(\theta)}\right) .
$$

The imaginary part of the integrand integrates to zero, because of the symmetry. We first look at

$$
\psi_{\boldsymbol{n}}(r, \boldsymbol{w}):=\int_{-n_{2}^{\prime} \pi}^{n_{2}^{\prime} \pi} \frac{D_{n_{2}, s_{2}, t_{2}}\left(\theta_{2} / n_{2}^{\prime}\right)}{n_{2}^{2}} \frac{g\left(\left(n^{*} r w_{2}\right)^{-1 / \alpha_{2}}, \theta_{2} / n_{2}^{\prime}\right)}{\left(n^{*}\right)^{1 / 2}} \mathbf{1}_{\Omega_{n}^{0,0}} d \theta_{2} .
$$

One can show by finding upper and lower bounds of $g$ depending on $\delta$ but not $\theta$,

$$
\lim _{\delta \downarrow 0} \limsup _{n \rightarrow \infty} \sup _{n^{*} r \boldsymbol{w} \in T_{\alpha}((0, \delta])}\left|\psi_{\boldsymbol{n}}(r, \boldsymbol{w})-s_{2} t_{2} \pi\right|=0 .
$$

From now on, write

$$
\rho=w_{1} \delta \wedge w_{2} \delta^{\alpha_{2}} .
$$

Introduce

$$
\Psi_{n_{1}}^{ \pm}(\delta):=\frac{1}{\log n_{1}} \int_{\Delta_{1}} \int_{-n_{1} \pi}^{n_{1} \pi} \int_{\left(n_{1} \rho\right)^{-1}}^{\infty} \frac{D_{n_{1}, s_{1}, t_{1}}^{ \pm}\left(\theta_{1} / n_{1}\right)}{n_{1}^{2}} \frac{g\left(\left(n_{1} r w_{1}\right)^{-1}, \theta_{1} / n_{1}\right)}{n_{1}} \frac{d r}{r^{2}} d \theta_{1} \Lambda\left(d w_{1}\right) .
$$


We shall treat the positive and negative parts of $D$, denoted by $D^{ \pm}$, separately, and we accordingly let $\mathfrak{D}_{s_{1}, t_{1}}^{ \pm}\left(\theta_{1}\right)$ denote the positive/negative part of the real part of $\left(e^{i s_{1} \theta_{1}}-\right.$ $1) \overline{\left(e^{i t_{1} \theta_{1}}-1\right)} /\left|\theta_{1}\right|^{2}$. We shall show

$$
\begin{aligned}
(2-\delta) \sigma_{ \pm}^{2} \leq & \liminf _{n_{1} \rightarrow \infty} \Psi_{n_{1}}^{ \pm}(\delta) \leq \limsup _{n_{1} \rightarrow \infty} \Psi_{n_{1}}^{ \pm}(\delta) \leq 2 \sigma_{ \pm}^{2} \\
& \text { with } \sigma_{ \pm}^{2}:=\int_{\Delta_{1}} w_{1} \Lambda(d \boldsymbol{w}) \int_{\mathbb{R}} \mathfrak{D}_{s_{1}, t_{1}}^{ \pm}\left(\theta_{1}\right) d \theta_{1} \text { and for all } \delta \in(0,1)
\end{aligned}
$$

from which, and thanks to (7.4), the desired result follows. We only prove the above for $\Psi_{n_{1}}^{+}(\delta)$, as the proof for $\Psi_{n_{1}}^{-}(\delta)$ is identical.

To simplify further the notation, introduce

$$
\widetilde{g}_{n_{1}}\left(r, \theta_{1}, w_{1}\right):=\frac{r w_{1}^{-1}\left(2-r\left(n_{1} w_{1}\right)^{-1}\right)}{r^{2} w_{1}^{-2}+2\left(1-r\left(n_{1} w_{1}\right)^{-1}\right) n_{1}^{2}\left(1-\cos \left(\theta_{1} / n_{1}\right)\right)} .
$$

By change of variable $r \rightarrow r^{-1}$, we rewrite (7.5) as

$$
\begin{aligned}
\Psi_{n_{1}}^{+}(\delta) & =\frac{1}{\log n_{1}} \int_{\Delta_{1}} \int_{-n_{1} \pi}^{n_{1} \pi} \int_{0}^{n_{1} \rho} \frac{D_{n_{1}, s_{1}, t_{1}}^{+}\left(\theta_{1} / n_{1}\right)}{n_{1}^{2}} \widetilde{g}_{n_{1}}\left(r, \theta_{1}, w_{1}\right) d r d \theta_{1} \Lambda(d \boldsymbol{w}) \\
& =\frac{1}{\log n_{1}} \int_{\Delta_{1}} \int_{\mathbb{R}} \int_{0}^{n_{1} \rho} f_{n_{1}}\left(r, \theta_{1}, w_{1}\right) \mathbf{1}_{\left\{\theta_{1} \leq n_{1} \pi\right\}} d r d \theta_{1} \Lambda(d \boldsymbol{w}),
\end{aligned}
$$

break the triple integral above into two parts

$$
\int_{\Delta_{1}} \int_{-n_{1} \pi}^{n_{1} \pi} \int_{1}^{n_{1} \rho} f_{n_{1}} d r d \theta_{1} \Lambda(d \boldsymbol{w}) \quad \text { and } \quad \int_{\Delta_{1}} \int_{-n_{1} \pi}^{n_{1} \pi} \int_{0}^{1} f_{n_{1}} d r d \theta_{1} \Lambda(d \boldsymbol{w})
$$

and treat them separately. Asymptotically, the former contributes and the latter is negligible.

For the first integral in (7.7), by

$$
\widetilde{g}_{n_{1}}\left(r, \theta_{1}, w_{1}\right) \mathbf{1}_{\left\{r \in\left(0, n_{1} \rho\right]\right\}} \leq \frac{2 w_{1}}{r},
$$

we have

$$
\begin{aligned}
\frac{1}{\log n_{1}} \int_{\Delta_{1}} \int_{\mathbb{R}} \int_{1}^{n_{1} \rho} f_{n_{1}}\left(r, \theta_{1}, w_{1}\right) \mathbf{1}_{\left\{\left|\theta_{1}\right| \leq n_{1} \pi\right\}} d r d \theta_{1} \Lambda(d \boldsymbol{w}) \\
\quad \leq 2 \int_{\Delta_{1}} w_{1} \Lambda(d \boldsymbol{w}) \int_{\mathbb{R}} \frac{D_{n_{1}}^{+}\left(\theta_{1} / n_{1}\right)}{n_{1}^{2}} \mathbf{1}_{\left\{\left|\theta_{1}\right| \leq n_{1} \pi\right\}} d \theta_{1} .
\end{aligned}
$$

Therefore,

$$
\limsup _{n_{1} \rightarrow \infty} \frac{1}{\log n_{1}} \int_{\Delta_{1}} \int_{-n_{1} \pi}^{n_{1} \pi} \int_{1}^{n_{1} \rho} f_{n_{1}}\left(r, \theta_{1}, w_{1}\right) d r d \theta_{1} \Lambda(d \boldsymbol{w}) \leq 2 \sigma_{+}^{2} .
$$

imsart-bj ver. 2014/10/16 file: aggregation20190726BEJ_combined.tex date: July 29, 2019 
For a lower bound, remark that

$$
\widetilde{g}_{n_{1}}\left(r, \theta_{1}, w_{1}\right) \geq \frac{(2-\delta) w_{1} r}{r^{2}+C \theta_{1}^{2} w_{1}^{2}} \text { for all } r \in\left(0, n_{1} \rho\right] .
$$

We have, for $n_{1} \rho \geq 1$,

$$
\begin{aligned}
\int_{1}^{n_{1} \rho} \widetilde{g}_{n_{1}}\left(r, \theta_{1}, w_{1}\right) d r & \geq \frac{2-\delta}{2} w_{1}\left[\log \left(n_{1} \rho\right)^{2}-\log \left(1+C \theta_{1}^{2} w_{1}^{2}\right)\right] \\
& \geq(2-\delta) w_{1}\left(\log n_{1}-C\left(1+\left|\log w_{1}\right|+\left|\log w_{2}\right|+|\log \delta|+|\log | \theta_{1}||\right),\right.
\end{aligned}
$$

where in the last step we used inequalities $\log \left(n_{1} \rho\right) \geq \log n_{1}-|\log \rho| \geq \log n_{1}-$ $2\left(\left|\log w_{1}\right|+\left|\log w_{2}\right|+|\log \delta|\right)$ and $\log \left(1+C \theta_{1}^{2} w_{1}^{2}\right) \leq \log 2+\left|\log \left(2 C \theta_{1}^{2} w_{1}^{2}\right)\right| \leq C(1+$ $\left.|\log | \theta_{1}||+\left|\log w_{1}\right|\right)$.

Since (7.9) does not hold uniformly in $n_{1}$ for all $\boldsymbol{w} \in \Delta_{1}$, we cannot apply it directly when integrating with respect to $\Lambda(d \boldsymbol{w})$. Instead, we first apply the above to the integral restricted to $\left\{\boldsymbol{w} \in \Delta_{1}, w_{1} \in[\epsilon, 1-\epsilon]\right\}$, and then let $\epsilon \downarrow 0$. Eventually we obtain

$$
\liminf _{n_{1} \rightarrow \infty} \frac{1}{\log n_{1}} \int_{\Delta_{1}} \int_{-n_{1} \pi}^{n_{1} \pi} \int_{1}^{n_{1} \rho} f_{n_{1}}\left(r, \theta_{1}, w_{1}\right) d r d \theta_{1} \Lambda(d \boldsymbol{w}) \geq(2-\delta) \sigma_{+}^{2} .
$$

In this step, we need the assumption (7.2).

Next, to deal with the second integral in (7.7), we have, using the upper bound on $D$,

$$
\begin{aligned}
& \int_{-n_{1} \pi}^{n_{1} \pi} \int_{0}^{1} f_{n_{1}}\left(r, \theta_{1},\right.\left.w_{1}\right) d r d \theta_{1} \\
& \leq C \int_{0}^{1} \int_{-n_{1} \pi}^{n_{1} \pi} \min \left\{1, \frac{1}{\left|\theta_{1}\right|^{2}}\right\} \frac{r w_{1}}{r^{2}+C n_{1}^{2}\left(1-\cos \left(\theta_{1} / n_{1}\right)\right) w_{1}^{2}} d \theta_{1} d r .
\end{aligned}
$$

By restricting the inner integration to $\left\{\theta_{1} \in \mathbb{R}:\left|\theta_{1}\right| \leq n_{1} \delta\right\}$ and $\left\{\theta_{1} \in \mathbb{R}: n_{1} \delta<\left|\theta_{1}\right| \leq\right.$ $\left.n_{1} \pi\right\}$, and bounding $n_{1}^{2}\left(1-\cos \left(\theta_{1} / n_{1}\right)\right)$ from below by $C_{\delta} \theta_{1}^{2}$ and $C_{\delta}$, respectively, one eventually has

$$
\int_{\Delta_{1}} \int_{-n_{1} \pi}^{n_{1} \pi} \int_{0}^{1} f_{n_{1}} d r d \theta_{1} \Lambda(d \boldsymbol{w}) \leq C_{\delta}\left(1+1 / n_{1}\right) \int_{\Delta_{1}} w_{1} \Lambda(d \boldsymbol{w}) .
$$

Combining (7.8), (7.10) and (7.11), we have proved (7.6) and hence the desired result. 